\title{
Entropic Repulsion of the Lattice Free Field
}

\author{
Erwin Bolthausen, Jean-Dominique Deuschel, Ofer Zeitouni \\ Universität Zürich, Technische Universität Berlin and Technion, D-10623 Berlin, Germany
}

Received: 28 June 1994/in revised form: 31 October 1994

\begin{abstract}
Consider the massless free field on the $d$-dimensional lattice $\mathbb{Z}^{d}, d \geqq 3$; that is the centered Gaussian field on $\mathbb{R}^{\mathbb{Z}^{d}}$ with covariances given by the Green function of the simple random walk on $\mathbb{Z}^{d}$. We show that the probability, that all the spins are positive in a box of volume $N^{d}$, decays exponentially at a rate of order $N^{d-2} \log N$ and compute explicitly the corresponding constant in terms of the capacity of the unit cube. The result is extended to a class of transient random walks with transition functions in the domain of the normal and $\alpha$-stable law.
\end{abstract}

\section{Introduction and Result}

Let $Q=\left\{Q(k, j), k, j \in \mathbb{Z}^{d}\right\}$ be the transition matrix of a symmetric transient random walk on the $d$-dimensional lattice $\mathbb{Z}^{d}$. More specifically we will be interested in two types of situations:

(a) $d \geqq 3, Q$ is the transition function of the simple random walk:

$$
Q(i, k)= \begin{cases}\frac{1}{2 d} & \text { if }|i-k|=1, \\ 0 & \text { otherwise }\end{cases}
$$

(b) $d \geqq 1, q_{\alpha}$ is the density of the symmetric isotropic $\alpha$-stable law on $\mathbb{R}^{d}$ for some $0<\alpha<2 \wedge d$, see (A.1),

$$
Q(i, k)=\int_{V} q_{\alpha}\left(x+(i-k)^{+}\right) d x,
$$

where $V=\left[-\frac{1}{2}, \frac{1}{2}\right]^{d},(j)^{+}=\left(\left|j_{1}\right|,\left|j_{2}\right|, \ldots,\left|j_{d}\right|\right)$.

Let $G=\sum_{n=0}^{\infty} Q^{n}$ be the corresponding Green function. Then it is well known that

$$
\lim _{|k-\jmath| \rightarrow \infty} \frac{G(j, k)}{g_{\alpha}(j-k)}=1,
$$

The authors acknowledge support from the Swiss National Science Foundation, grant 21-29833.90 This research was partially supported by the foundation for promotion of research at the Technion. 
where $g_{\alpha}(x)=\omega_{\alpha, d}|x|^{-d+\alpha}$ is the Riesz kernel, $\omega_{\alpha, d}$ is a normalizing constant, cf. [0] and Lemma A.2, below. (In case (a), we set $\alpha=2$.)

The object of this paper will be the stationary centered Gaussian field $\{X(j)\}_{j \in \mathbb{Z}^{d}}$ of law $P$ on $\Omega=\mathbb{R}^{\mathbb{Z}^{d}}$ with covariances $G$. The relation between the transition matrix $Q$ and the Gaussian field $P$ is best explained by the following Gibbsian description of $P$ : let $P_{k}\left(\cdot \mid \mathscr{F}_{\{k\}} \mathrm{C}\right)$ be the conditional distribution of $X(k)$ given $\mathscr{F}_{\{k\}} \mathrm{C}=\sigma(X(j): j \neq k)$, then

$$
P_{k}\left(\cdot \mid \mathscr{F}_{\{k\}} \mathbf{C}\right)=\mathscr{N}(\tilde{X}(k) ; 1) \quad \text { with } \quad \tilde{X}(k)=\sum_{j \neq k} Q(k, j) X(j),
$$

where $\mathscr{N}\left(a ; \sigma^{2}\right)$ denotes the normal distribution with mean $a$ and variance $\sigma^{2}$, cf. pages $262-263$ of [8]. In particular, case (a) corresponds to a Markovian field, known in the literature as the (discrete) massless free field. Let $V_{N}=\left\{k \in \mathbb{Z}^{d}: \frac{k}{N} \in\right.$ $V\}$; the aim of this paper is to prove the following.

Theorem 1.1. Let $\mathbf{G}=G(0,0)$ and $\mathbf{C}=\operatorname{cap}_{\alpha}(V)$ be the capacity associated with $g_{\alpha}: \operatorname{cap}_{\alpha}(V)=\sup \left\{2 \mu(V)-\int_{V} \int_{V} g_{\alpha}(x-y) \mu(d x) \mu(d y): \mu\right.$ positive Radon measure on $V\}$, then

$$
\lim _{N \rightarrow \infty} \frac{1}{N^{d-x} \log N} \log P\left(X(k) \geqq 0 \quad \text { for } k \in V_{N}\right)=-\alpha \mathbf{G C} .
$$

Theorem 1.1 answers a question raised for the case (a) by Lebowitz and Maes on page 47 of [11], where they prove a decay of the order $\exp \left(-o\left(N^{d}\right)\right)$ and suggest the order $\exp \left(-O\left(N^{d-2}\right)\right.$ ) (see also [6] for related questions dealing with quasi-locality of the field $\left.\left\{\sigma(k)=\operatorname{sign}(X(k)): k \in \mathbb{Z}^{d}\right\}\right)$.

Actually we will prove a slightly more general result: let $\left\{b_{N}: N \in \mathbb{N}\right\} \subseteq \mathbb{R}$ be such that

$$
\lim _{N \rightarrow \infty} \frac{b_{N}}{\sqrt{\log N}}=b \in(-\sqrt{2 \alpha \mathbf{G}}, \infty)
$$

then

$$
\lim _{N \rightarrow \infty} \frac{1}{N^{d-\alpha} \log N} \log P\left(X(k) \geqq b_{N} \quad \text { for } k \in V_{N}\right)=-(\sqrt{2 \alpha \mathbf{G}}+b)^{2} \frac{\mathbf{C}}{2} .
$$

Also, (a) and (b) can be generalized to

$\left(\mathrm{a}^{\prime}\right) d \geqq 3, Q(i, k)=Q(k, i)=Q(i-k, 0) \geqq 0$ is irreducible and of finite range $R \geqq 1$ :

$$
Q(i, k)=0, \quad|i-k|>R .
$$

$\left(\mathrm{b}^{\prime}\right) d \geqq 1, Q(i, k)=Q(k, i)=Q(i-k, 0) \geqq 0$ is strongly aperiodic and satisfies

$$
\lim _{|k-j| \rightarrow \infty}|k-j|^{d+\alpha} Q(k, j)=c_{\alpha}>0 .
$$

Of course, in case $\left(\mathrm{a}^{\prime}\right), g_{2}$ and $\mathrm{cap}_{2}$ have to be adapted to the corresponding kernel and capacity, cf. (0.6) and (0.9) of [0].

The presence of the $\log N$ factor in the exponent of Theorem 1.1 is best explained by the fact, that, under the condition

$$
\Omega_{N} \equiv\left\{X(k) \geqq 0 \text { for } k \in V_{N}\right\},
$$

most of the $X(k), k \in V_{N}$, will be at the level $\sqrt{2 \alpha \mathbf{G} \log N}$ (see also [1]). 
Proposition 1.3. Let $a<2 \alpha \mathbf{G}, b>2 \alpha \mathbf{G}$ and $\varepsilon>0$, then

$$
\lim _{N \rightarrow \infty} \sup _{k \in V_{N, \varepsilon}} P\left(X(k) \leqq \sqrt{a \log N} \mid \Omega_{N}\right)=0,
$$

and

$$
\lim _{N \rightarrow \infty} \sup _{k \in V_{N, \varepsilon}} P\left(X(k) \geqq \sqrt{b \log N} \mid \Omega_{N}\right)=0,
$$

where $V_{N, \varepsilon}=\left\{k \in V_{N}\right.$ : $\left.\operatorname{dist}\left(k, V_{N}^{\mathbf{C}}\right) \geqq \varepsilon N\right\}$.

Proposition 1.3 suggests that, under the conditioning $\Omega_{N}$, the field $P$ converges weakly to $P_{N}$, the stationary Gaussian field with mean $\sqrt{2 \alpha \mathbf{G} \log N}$, that is

$$
P\left(\cdot-\sqrt{2 \alpha \mathbf{G} \log N} \mid X(k) \geqq 0 \quad \text { for } k \in V_{N}\right) \Rightarrow P(\cdot) .
$$

This is connected with the so-called entropic repulsion. The long range correlations make the field relatively stiff, but the local fluctuations push the random "surface" to infinity in the presence of a hard wall, i.e. the conditioning that the fields stays positive on $V_{N}$.

Theorem 1.1 is closely related to the theory of large deviations. More precisely, let $\mathscr{M}_{1}(\mathbb{R})$ be the set of probability distributions on $\mathbb{R}$ endowed with the weak topology and set $\mathscr{A}=\left\{v \in \mathscr{M}_{1}(\mathbb{R}): v([0, \infty))=1\right\}$. $\mathscr{A}$ is a closed set with empty interior. Next let

$$
L_{V_{N}}=\frac{1}{\left|V_{N}\right|} \sum_{k \in V_{N}} \delta_{X(k)} \in \mathscr{M}_{1}(\mathbb{R})
$$

be the empirical distribution of the box $V_{N}$, then

$$
\Omega_{N}=\left\{L_{V_{N}} \in \mathscr{A}\right\} .
$$

Using the $N^{d-2}$ large deviation principle derived for $P \circ\left(L_{V_{N}}\right)^{-1}$ in case $\left(\mathrm{a}^{\prime}\right)$, Theorem 0.10 of $[0]$, one sees that

$$
\limsup _{N \rightarrow \infty} \frac{1}{N^{d-2}} \log P\left(\Omega_{N}\right)=\limsup _{N \rightarrow \infty} \frac{1}{N^{d-2}} \log P\left(L_{V_{N}} \in \mathscr{A}\right) \leqq-\infty,
$$

since the corresponding rate function is infinite on $\mathscr{A}$. Unlike the weakly dependent case (see below), Theorem 1.1 cannot be proved by standard large deviation techniques.

The proof of Theorem 1.1 is divided into two parts. The lower bound based on a conditioning and entropy argument is given in Sect. 2, the upper bound in Sect. 3. Here we follow a conditioning argument as in the proof of the upper bound in [0]. Proposition 1.3 is proved in Sect. 4.

We conclude this section with a quick survey of the weakly dependent situation with fast decaying covariances. More precisely, let $0<\varepsilon<1$ and consider the Green function $G^{\varepsilon}$ of the random walk with constant killing probability $\varepsilon$ :

$$
G^{\varepsilon}=\sum_{n=0}^{\infty}(1-\varepsilon)^{n} Q^{n}
$$

Next, let $P^{\varepsilon}$ be the centered Gaussian field with covariance $G^{\varepsilon}$, (in case (a) the so-called discrete free field with positive mass $\varepsilon$ ). $P^{\varepsilon}$ is hypercontractive and 
$P^{\varepsilon} \circ\left(L_{V_{N}}\right)^{-1}$ satisfies a volume order large deviation principle with the good rate function $\mathbf{h}\left(\cdot \mid P^{\varepsilon}\right)$, the specific entropy, cf. [0]. Let $\mathscr{M}_{1}^{S}(\Omega)$ be the set of stationary probability measures on $\Omega$ and denote by $\Pi: \mathscr{M}_{1}^{S}(\Omega) \rightarrow \mathscr{M}_{1}(\mathbb{R})$ the projection to the one dimensional coordinate.

Proposition 1.7. Assume $\left(\mathrm{a}^{\prime}\right)$. There exists a unique $Q^{*} \in \mathscr{M}_{1}^{S}(\Omega)$ with $\Pi\left(Q^{*}\right) \in \mathscr{A}$ such that

$$
\mathbf{h}\left(Q^{*} \mid P^{\varepsilon}\right)=\inf \left\{\mathbf{h}\left(Q \mid P^{\varepsilon}\right): Q \in \mathscr{M}_{1}^{S}(\Omega), \Pi(Q) \in \mathscr{A}\right\} \in \mathbb{R}^{+}
$$

and

$$
\lim _{N \rightarrow \infty} \frac{1}{\left|V_{N}\right|} \log P^{\varepsilon}\left(\Omega_{N}\right)=-\mathbf{h}\left(Q^{*} \mid P^{\varepsilon}\right) .
$$

Moreover, $P^{\varepsilon}\left(\cdot \mid \Omega_{N}\right)$ converges weakly to $Q^{*}$ as $N \rightarrow \infty$.

The proof of Proposition 1.7 together with the Gibbsian characterization of $Q^{*}$ is given at the end of Sect. 3 .

\section{Proof of the Lower Bound}

The aim of this section is to prove the following lower bound:

Proposition 2.1. Let $\left\{b_{N}: N \in \mathbb{N}\right\}$ satisfy (1.2) and set $\tilde{\Omega}_{N} \equiv\left\{X(k) \geqq b_{N}\right.$ for $\left.k \in V_{N}\right\}$. Then, under $\left(\mathrm{a}^{\prime}\right)$ or $\left(\mathrm{b}^{\prime}\right)$,

$$
\liminf _{N \rightarrow \infty} \frac{1}{N^{d-x} \log N} \log P\left(\tilde{\Omega}_{N}\right) \geqq-(\sqrt{2 \alpha \mathbf{G}}+b)^{2} \frac{\mathbf{C}}{2} .
$$

We will always be working under $\left(a^{\prime}\right)$ or $\left(b^{\prime}\right)$. As a warming up we start with a simpler result which misses the correct constant but illustrates quite well the essence of the argument

\section{Lemma 2.3.}

$$
\liminf _{N \rightarrow \infty} \frac{1}{N^{d-\alpha} \log N} \log P\left(\Omega_{N}\right) \geqq-d \mathbf{G C} .
$$

Proof. For any $a>2 d \mathbf{G}$ let $P_{N}$ be the Gaussian field on $\Omega$ with mean $\sqrt{a \log N}$ and covariance $G$. Let $\mathscr{F}_{V_{N}}=\sigma\left(X(k): k \in V_{N}\right)$ and set $F_{N}=\left.\frac{d P_{N}}{d P}\right|_{\mathscr{F}} V_{N}$. Then

$$
\mathbf{H}_{N}\left(P_{N} \mid P\right) \equiv \int_{\Omega} \log F_{N} F_{N} d P=\frac{a \log N}{2}\left\langle 1_{V_{N}}, G_{N}^{-1} 1_{V_{N}}\right\rangle_{V_{N}},
$$

where $\langle\cdot, \cdot\rangle_{V_{N}}$ is the $L^{2}\left(V_{N}\right)$-scalar product, $G_{N}$ is the covariance matrix restricted to $V_{N}$ and $G_{N}^{-1}$ is the inverse of $G_{N}$ (beware that $\left(G^{-1}\right)_{N} \neq G_{N}^{-1}$ !) Note that $\operatorname{cap}_{N}\left(V_{N}\right) \equiv\left\langle 1_{V_{N}}, G_{N}^{-1} 1_{V_{N}}\right\rangle_{V_{N}}$ is the capacity of $V_{N}$ with respect to the random walk generated by $Q$, cf. Sect. 25 of [13]. We have

$$
\lim _{N \rightarrow \infty} \frac{1}{N^{d-\alpha}} \operatorname{cap}_{N}\left(V_{N}\right)=\operatorname{cap}_{\alpha}(V)=\mathbf{C} \text {. }
$$


In case $\left(\mathrm{a}^{\prime}\right)$, this is proved in Lemmas 2.1 and 2.2 of [0]. We give a proof of (2.5) for $0<\alpha<2 \wedge d$ in Proposition A.11 below. Thus

$$
\lim _{N \rightarrow \infty} \frac{1}{N^{d-x} \log N} \mathbf{H}_{N}\left(P_{N} \mid P\right)=\frac{a \mathbf{C}}{2} .
$$

Also we have

$$
\begin{aligned}
P_{N}\left(\Omega_{N}^{\mathbf{C}}\right) & =P_{N}\left(\bigcup_{k \in V_{N}}\{X(k)<0\}\right) \leqq\left|V_{N}\right| P_{N}(X(k)<0) \\
& =N^{d} P(X(k)<-\sqrt{a \log N})=N^{d} \phi(-\sqrt{a \log N / \mathbf{G}})
\end{aligned}
$$

where $\phi(x) \equiv(2 \pi)^{-1 / 2} \int_{-\infty}^{x} e^{-t^{2} / 2} d t \leqq \frac{1}{2} e^{-x^{2} / 2}$ for $x \leqq 0$. Thus

$$
P_{N}\left(\Omega_{N}^{\mathbf{C}}\right) \leqq \frac{1}{2} N^{d-\frac{a}{2 G}} \longrightarrow 0
$$

as $N \rightarrow \infty$ and therefore

$$
\lim _{N \rightarrow \infty} P_{N}\left(\Omega_{N}\right)=1 .
$$

Now (2.4) follows from (2.6) and (2.7) by the usual change of measure argument: since $x \longrightarrow \log x$ is concave, we have by Jensen's inequality

$$
\begin{aligned}
\log \frac{P\left(\Omega_{N}\right)}{P_{N}\left(\Omega_{N}\right)} & =\log \int_{\Omega_{N}} F_{N}^{-1} \frac{d P_{N}}{P_{N}\left(\Omega_{N}\right)} \geqq \int_{\Omega_{N}} \log F_{N}^{-1} \frac{d P_{N}}{P_{N}\left(\Omega_{N}\right)} \\
& =-\frac{1}{P_{N}\left(\Omega_{N}\right)} \int_{\Omega_{N}} \log F_{N} F_{N} d P \geqq-\frac{1}{P_{N}\left(\Omega_{N}\right)}\left(\int_{\Omega} \log F_{N} F_{N} d P+e^{-1}\right) \\
& =-\frac{1}{P_{N}\left(\Omega_{N}\right)}\left(\mathbf{H}_{N}\left(P_{N} \mid P\right)+e^{-1}\right)
\end{aligned}
$$

where in the last inequality we have used the fact that $x \longrightarrow x \log x \geqq-e^{-1}$. Taking the lim inf on both sides, we get (2.4) by (2.6) and (2.7).

The major obstacle in getting the correct constant for the lower bound with the above method, is the rather poor estimate of $P_{N}\left(\Omega_{N}^{\mathbf{C}}\right)$ which forces $a>2 d \mathbf{G}$. In order to overcome this difficulty and prove Proposition 2.1 , let us consider the auxiliary centered Gaussian field $\left\{X(i, a): i \in \mathbb{Z}^{d}, a \in\{1, \ldots, L\}\right\}$ with covariances

$$
E[X(i, a) X(j, b)]= \begin{cases}\frac{G(i, j)}{L} & i \neq j \\ \frac{G(0,0)-1}{L}+\delta_{a, b} & i=j .\end{cases}
$$

Remark that the covariance matrix is also the Green function of a random walk $\left\{\xi_{0}, \xi_{1}, \ldots\right\}$ with transition probabilities

$$
Q((i, a),(j, b))=\frac{Q(i, j)}{L} .
$$


We denote by $\mathbb{P}_{(i, a)}$ the law of this random walk with start $\xi_{0}$ at $(i, a)$. Let

$$
Y(i) \equiv \frac{1}{\sqrt{L}} \sum_{a=1}^{L} X(i, a) \quad Z(i) \equiv X(i, 1),
$$

and set $\mathscr{F}_{Z} \equiv \sigma\left(Z(i), i \in \mathbb{Z}^{d}\right)$. Note that $E[Y(i) Y(j)]=G(i, j)$, thus

$$
\mathscr{L}\left(\{Y(i)\}_{i \in \mathbb{Z}^{d}}\right)=\mathscr{L}\left(\{X(i)\}_{i \in \mathbb{Z}^{d}}\right) .
$$

We calculate the conditional law of $Y$ given $\mathscr{F}_{Z}$ following the technique of the Appendix of [0]: Let $\tau \equiv \inf \left\{n \geqq 0: \xi_{n} \in \mathbb{Z}^{d} \times\{1\}\right\}$ and

$$
q^{L}((i, a), j)=\mathbb{P}_{(i, a)}\left(\xi_{\tau}=(j, 1)\right) .
$$

Obviously $q^{L}((i, 1), j)=\delta_{i, J}$, and, for $a \geqq 2, q^{L}((i, a), j)$ does not depend on $a$. We denote its value by $\tilde{q}^{L}(i, j)$. By the random walk representation

$$
E\left[X(i, a) \mid \mathscr{F}_{Z}\right]=\sum_{j \in \mathbb{Z}^{d}} q^{L}((i, a), j) Z(j) .
$$

Next, let $\eta(i) \equiv E\left[Y(i) \mid \mathscr{F}_{Z}\right]$, then

$$
\eta(i)=\frac{1}{\sqrt{L}} \sum_{j \in \mathbb{Z}^{d}} \sum_{a=1}^{L} q^{L}((i, a), j) Z(j)=\frac{L-1}{\sqrt{L}} \sum_{j \in \mathbb{Z}^{d}} \tilde{q}^{L}(i, j) Z(j)+\frac{1}{\sqrt{L}} Z(i) .
$$

The covariances of $Z$ are given by

$$
G_{Z}(i, j)= \begin{cases}\frac{G(i, j)}{L} & i \neq j \\ \left(1-\frac{1}{L}\right)+\frac{G(0,0)}{L} & i=j .\end{cases}
$$

We can represent the $Z$-field as

$$
Z(i)=\frac{U(i)}{\sqrt{L}}+\sqrt{\frac{L-1}{L}} V(i),
$$

where $\mathscr{L}(U(\cdot))=\mathscr{L}(Y(\cdot))$ and the $\{V(i)\}$ are i.i.d. $\mathscr{N}(0 ; 1)$, independent of $\{U(i)\}$. Thus

$$
\begin{aligned}
\eta(i)= & \left(1-\frac{1}{L}\right) \sum_{j \in \mathbb{Z}^{d}} \tilde{q}^{L}(i, j) U(j) \\
& +\frac{1}{L} U(i)+\frac{(L-1)^{3 / 2}}{L} \sum_{j \in \mathbb{Z}^{d}} \tilde{q}^{L}(i, j) V(j)+\frac{\sqrt{L-1}}{L} V(i) .
\end{aligned}
$$

Lemma 2.9. $\sigma_{L}^{2} \equiv \operatorname{var}(\eta(i)) \rightarrow 0$ as $L \rightarrow \infty$.

Proof. The only problem is the first and third summation in the previous expression for $\eta(i)$ :

$$
\operatorname{var}\left(\sum_{j} \tilde{q}^{L}(i, j) U(j)\right)=\sum_{j, k} \tilde{q}^{L}(i, j) G(j, k) \tilde{q}^{L}(k, i) \rightarrow 0 \quad \text { as } L \rightarrow \infty,
$$


and

$$
\operatorname{var}\left(L^{1 / 2} \sum_{j \in \mathbb{Z}^{d}} \tilde{q}^{L}(i, j) V(j)\right)=L \sum_{j \in \mathbb{Z}^{d}}\left(\tilde{q}^{L}(i, j)\right)^{2} \rightarrow 0 \quad \text { as } L \rightarrow \infty .
$$

Note that, starting at any $a \geqq 2, \xi_{n}$ is a random walk until the first (geometrically distributed, independent) time in which $a=1$ is hit. Let $\left\{\zeta_{n}\right\}$ and $\left\{\zeta_{n}^{\prime}\right\}$ be independent random walks on $\mathbb{Z}^{d}$ generated by $Q$, and let $\tau, \tau^{\prime}$ be independent random variables, independent of $\left\{\zeta_{n}\right\},\left\{\zeta_{n}^{\prime}\right\}$, with geometric distribution of parameter $\frac{1}{L}$. Let $\mathbb{P}_{0}$ denote the joint law of $\tau, \tau^{\prime},\left\{\zeta_{n}\right\},\left\{\zeta_{n}^{\prime}\right\}$. Then

$$
\tilde{q}^{L}(i, j)=\mathbb{P}_{(i, a)}\left(\xi_{\tau}=(j, 1)\right)=\mathbb{P}_{0}\left(\zeta_{\tau}=j-i\right)
$$

and

$$
\begin{aligned}
\sum_{j \in \mathbb{Z}^{d}}\left(\tilde{q}^{L}(i, j)\right)^{2} & =\sum_{j \in \mathbb{Z}^{d}} \mathbb{P}_{0}\left(\zeta_{\tau}=j\right) \mathbb{P}_{0}\left(\zeta_{\tau^{\prime}}^{\prime}=j\right) \\
& =\mathbb{P}_{0}\left(\zeta_{\tau}=\zeta_{\tau^{\prime}}^{\prime}\right)=\mathbb{P}_{0}\left(\zeta_{\tau+\tau^{\prime}}=0\right) \\
& =\sum_{n, n^{\prime}=1}^{\infty} \mathbb{P}_{0}\left(\zeta_{n+n^{\prime}}=0\right) \mathbb{P}_{0}(\tau=n) \mathbb{P}_{0}\left(\tau=n^{\prime}\right)
\end{aligned}
$$

Further we have $\mathbb{P}_{0}\left(\zeta_{n+n^{\prime}}=0\right) \leqq c\left(n+n^{\prime}\right)^{-d / \alpha}$, for some $c>0$ (this follows from local central limit theorem, cf. [13], Sect. 26 in case $\left(a^{\prime}\right)$, and Lemma A.1 below in case $\left.\left(\mathrm{b}^{\prime}\right)\right)$, and $\mathbb{P}_{0}(\tau=n) \leqq \frac{K}{L} e^{-n / L}$ for some $K>0$. This yields

$$
\mathbb{P}_{0}\left(\zeta_{\tau+\tau^{\prime}}=0\right) \leqq K^{2} c \sum_{n, n^{\prime}=1}^{\infty}\left(n+n^{\prime}\right)^{-d / \alpha} e^{-n / L} e^{-n^{\prime} / L} L^{-2} \leqq k^{\prime} \sum_{n=1}^{\infty} n^{-d / \alpha+1} e^{-n / L} L^{-2}
$$

for some $k^{\prime}>0$. The later is of order $L^{-2}$ for $0<\alpha<d / 2, L^{-2} \log L$ for $\alpha=d / 2$, and $L^{-d / \alpha}$ for $2 \wedge d \geqq \alpha>d / 2$. This proves (2.11). As for (2.10), note that

$$
\begin{aligned}
\sum_{j, k} \tilde{q}^{L}(i, j) G(j, k) \tilde{q}^{L}(k, i)= & \sum_{m=0}^{\infty} \sum_{j, k} \mathbb{P}_{i}\left(\zeta_{\tau}=j, \zeta_{\tau+m}=k, \zeta_{\tau+m+\tau^{\prime}}=i\right) \\
= & \sum_{m=0}^{\infty} \mathbb{P}_{i}\left(\zeta_{\tau+m+\tau^{\prime}}=i\right)=\sum_{n, n^{\prime}, m} \mathbb{P}_{0}\left(\zeta_{n+m+n^{\prime}}=0\right) \\
& \times \mathbb{P}_{0}(\tau=n) \mathbb{P}_{0}\left(\tau=n^{\prime}\right) \\
\leqq & K^{2} c \sum_{n, n^{\prime}, m}\left(n+n^{\prime}+m\right)^{-d / \alpha} e^{-n / L} e^{-n^{\prime} / L} L^{-2} \\
\leqq & K^{\prime} \sum_{n=1}^{\infty} n^{-d / \alpha+2} e^{-n / L} L^{-2}
\end{aligned}
$$

for some $K^{\prime}>0$. This is of order $L^{-2}$ for $0<\alpha<d / 3, L^{-2} \log L$ for $\alpha=d / 3$ and $L^{-d / \alpha+1}$ for $2 \wedge d \geqq \alpha>d / 3$. This shows (2.10).

Lemma 2.12. Let $x>0$, then

$$
\liminf _{L \rightarrow \infty} \liminf _{N \rightarrow \infty} \frac{1}{N^{d-\alpha} \log N} \log P\left(\eta(i) \geqq \sqrt{x \log N}, i \in V_{N}\right) \geqq-\frac{x \mathbf{C}}{2} .
$$


Proof. We follow the proof of Lemma 2.3: Choose $x^{\prime}>x$, let $\hat{P}$ denote the law of $(U(i), V(i))_{i \in \mathbb{Z}^{d}}$, and let $\hat{P}_{N}$ be the law of $(U(i), V(i))_{i \in \mathbb{Z}^{d}}$, where $U(i)$ has mean $\sqrt{x^{\prime} \log N}$ and $V(i)$ is unchanged. Then

$$
\lim _{N \rightarrow \infty} \frac{1}{N^{d-\alpha} \log N} \mathbf{H}_{N}\left(\hat{P}_{N} \mid \hat{P}\right)=\frac{x^{\prime} \mathbf{C}}{2} .
$$

Also $E_{\hat{P}_{N}}[\eta(i)]=\sqrt{x^{\prime} \log N}$, and

$$
\begin{aligned}
\hat{P}_{N}\left(\eta(i) \geqq \sqrt{x \log N}, i \in V_{N}\right) & =\hat{P}\left(\eta(i) \geqq \sqrt{\log N}\left(\sqrt{x}-\sqrt{x^{\prime}}\right), i \in V_{N}\right) \\
& \geqq 1-\left|V_{N}\right| \hat{P}\left(\eta(i)<-\sqrt{\log N}\left(\sqrt{x^{\prime}}-\sqrt{x}\right)\right) \\
& =1-\left|V_{N}\right| \phi\left(-\frac{\sqrt{\log N}\left(\sqrt{x^{\prime}}-\sqrt{x}\right)}{\sigma_{L}}\right) .
\end{aligned}
$$

If $L$ is so large that $\frac{\left(\sqrt{x^{\prime}}-\sqrt{x}\right)^{2}}{\sigma_{L}^{2}}>2 d$, we have $\lim _{N \rightarrow \infty}\left|V_{N}\right| \phi\left(-\frac{\sqrt{\log N}\left(\sqrt{x^{\prime}}-\sqrt{x}\right)}{\sigma_{L}}\right)=0$, cf. (2.7), and therefore

$$
\lim _{N \rightarrow \infty} \hat{P}_{N}\left(\eta(i) \geqq \sqrt{x \log N}, i \in V_{N}\right)=1 .
$$

We proceed from here as in the proof of Lemma 2.3.

Conditionally on $\mathscr{F}_{Z},(Y(i))_{i \in \mathbb{Z}^{d}}$ is Gaussian with mean $\eta(i)$. We need some information about the conditional covariances:

Lemma 2.13. Let

$$
G_{Z}^{L}(i, j)=E\left[(Y(i)-\eta(i))(Y(j)-\eta(j)) \mid \mathscr{F}_{Z}\right],
$$

then

$$
G_{Z}^{L}(i, j) \geqq 0
$$

and

$$
\mathbf{G}_{\mathbf{Z}}^{\mathbf{L}} \equiv G_{Z}^{L}(0,0) \rightarrow G(0,0)=\mathbf{G} \quad \text { as } L \rightarrow \infty .
$$

Proof. By the random walk representation

$$
\operatorname{cov}\left(X(i, a), X(j, b) \mid \mathscr{F}_{Z}\right)=\mathbb{E}_{(i, a)}\left[\sum_{n=0}^{\tau-1} 1_{\xi_{n}=(j, b)}\right],
$$

cf. Lemma A.6 of [0], which does not depend on the Z-field. Equation (2.14) is immediate. Next note that

$$
G_{Z}^{L}(i, j)=G(i, j)-\operatorname{cov}(\eta(i), \eta(j)),
$$

this implies (2.15) by Lemma 2.9 .

Proof of Proposition 2.1. For each $\delta>0$, let $N_{0}$ be such that $\frac{b_{N}}{\sqrt{\log N}}<b+\delta, N \geqq$ $N_{0}$. Choose $x>(\sqrt{2 \alpha \mathbf{G}}+b+\delta)^{2}$, then

$$
P\left(Y(i) \geqq b_{N}, i \in V_{N}\right) \geqq E\left[P\left(Y(i) \geqq b_{N}, i \in V_{N} \mid \mathscr{F}_{Z}\right) ; \eta(i) \geqq \sqrt{x \log N}, i \in V_{N}\right] .
$$


On $\left\{\eta(i) \geqq \sqrt{x \log N}, i \in V_{N}\right\}$,

$$
P\left(Y(i) \geqq b_{N}, i \in V_{N} \mid \mathscr{F}_{Z}\right) \geqq P\left(Y(i)-\eta(i) \geqq-\sqrt{x \log N}+b_{N}, i \in V_{N} \mid \mathscr{F}_{Z}\right) .
$$

Next, by (2.14), using Slepian's inequality,

$$
\begin{aligned}
P(Y(i)-\eta(i) & \left.\geqq-\sqrt{x \log N}+b_{N}, i \in V_{N} \mid \mathscr{F}_{Z}\right) \geqq \prod_{i \in V_{N}} P(Y(i)-\eta(i) \\
& \left.\geqq-\sqrt{x \log N}+b_{N} \mid \mathscr{F}_{Z}\right) \\
& =\left(1-\phi\left(-\left(\sqrt{x \log N}-b_{N}\right) / \sqrt{\mathbf{G}_{\mathbf{Z}}^{\mathbf{L}}}\right)\right)^{N^{d}} .
\end{aligned}
$$

Since $\mathbf{G}_{\mathbf{Z}}^{\mathbf{L}} \leqq \mathbf{G}, \frac{(\sqrt{x}-b-\delta)^{2}}{\mathbf{G}_{\mathbf{Z}}^{\mathbf{L}}} \geqq 2 \alpha+2 \varepsilon$ for some $\varepsilon>0$ independent of $L$. Thus for large $N \geqq N_{0}$,

$$
\begin{aligned}
P(Y(i)-\eta(i) & \left.\geqq-\sqrt{x \log N}+b_{N}, i \in V_{N} \mid \mathscr{F}_{Z}\right) \\
& \geqq\left(1-\frac{1}{2} \exp \left(-\frac{(\sqrt{x}-b-\delta)^{2} \log N}{2 \mathbf{G}_{\mathbf{Z}}^{\mathbf{L}}}\right)\right)^{N^{d}} \\
& \geqq e^{-\frac{1}{2}} N^{d-\alpha-\varepsilon} .
\end{aligned}
$$

In view of the above this shows

$$
P\left(Y(i) \geqq b_{N}, i \in V_{N}\right) \geqq e^{-\frac{1}{2} N^{d-\alpha-\varepsilon}} P\left(\eta(i) \geqq \sqrt{x \log N}, i \in V_{N}\right), \quad N \geqq N_{0} .
$$

Using Lemma 2.12 and the fact that $x>(\sqrt{2 \alpha \mathbf{G}}+b+\delta)^{2}$ and $\delta>0$ were arbitrary gives the claim.

\section{The Upper Bound}

In this section we give a proof of

Proposition 3.1. Let $\left\{b_{N}: N \in \mathbb{N}\right\}$ satisfy (1.2) and set $\tilde{\Omega}_{N}=\left\{X(k) \geqq b_{N}\right.$ for $\left.k \in V_{N}\right\}$. Assume $\left(\mathrm{a}^{\prime}\right)$ or $\left(\mathrm{b}^{\prime}\right)$, then

$$
\limsup _{N \rightarrow \infty} \frac{1}{N^{d-x} \log N} \log P\left(\tilde{\Omega}_{N}\right) \leqq-(\sqrt{2 \alpha \mathbf{G}}+b)^{2} \frac{\mathbf{C}}{2} .
$$

The major tool in the derivation of the upper bound, will be a conditioning argument on the lattice $L \mathbb{Z}^{d}$. Let $L \in 2 \mathbb{N}^{+}$be fixed and set

$$
\Lambda \equiv(L / 2, \ldots, L / 2)+L \mathbb{Z}^{d}, \quad \Lambda_{N}=\Lambda \cap V_{N} .
$$

Next let $\mathscr{F}_{L}=\sigma\left(X(i): i \in L \mathbb{Z}^{d}\right)$,

$$
\bar{X}(i)=E\left[X(i) \mid \mathscr{F}_{L}\right]=\sum_{j \in L \mathbb{Z}^{d}} q^{L}(i, j) X(j) \quad G^{L}(i, j)=\operatorname{cov}\left(X(i), X(j) \mid \mathscr{F}_{L}\right) .
$$

By the random walk representation, we have

$$
q^{L}(i, j)=\mathbb{P}_{i}\left(\xi_{\tau}=j\right), \quad G^{L}(i, j)=\mathbb{E}_{i}\left[\sum_{n=0}^{\tau-1} 1_{\xi_{n}=j}\right],
$$


where $\left\{\xi_{n}: n \in \mathbb{N}\right\}$ is a random walk generated by $Q$ and $\tau=\inf \left\{n \geqq 0: \xi_{n} \in L \mathbb{Z}^{d}\right\}$. In contrast to the original covariance $G, G^{L}$ is fast decaying. More precisely, in case $\left(\mathrm{a}^{\prime}\right)$ we have an exponential decay:

$$
G^{L}(i, j) \leqq c_{1} \exp \left(-c_{2}|i-j| L^{-d / 2}\right)
$$

for some $c_{1}, c_{2}>0$, cf. Lemma A.7 of [0], whereas in case $\left(\mathrm{b}^{\prime}\right)$, we have a fast algebraic decay:

$$
G^{L}(i, j) \leqq c_{3} L^{c_{4}}(\log |i-j|)^{d+2+\alpha}|i-j|^{-d-\alpha}, \quad|i-j|>1
$$

for some $c_{3}, c_{4}>0$, cf. Proposition A.10 in the Appendix. Also in both cases, if $\mathbf{G}^{\mathbf{L}} \equiv G^{L}(i, i), i \in \Lambda$, then

$$
\lim _{L \rightarrow \infty} \mathbf{G}^{\mathbf{L}}=\mathbf{G}
$$

Our first step is the following hypercontractive estimate

Lemma 3.5. There exists $K_{L}>0$ such that

$$
P\left(X(i) \geqq b_{N}, i \in \Lambda_{N} \mid \mathscr{F}_{L}\right) \leqq \prod_{i \in \Lambda_{N}} P\left(X(i) \geqq b_{N} \mid \mathscr{F}_{L}\right)^{K_{L}}
$$

Proof. The proof follows from Proposition A.18 below applied to the $\mathscr{F}_{L}$ conditioned field by using the function $\left.f(\cdot)=1_{\{} \cdot \geqq b_{N}\right\}$.

For fixed $\Delta \in \mathbb{N}$ consider a partition of $V_{N}$ into boxes $\left\{V_{N}^{i}: i \in V_{\Delta}\right\}$ of side $[N / \Delta]: V_{N}^{i}=V_{[N / \Delta]}+i[N / \Delta]$ and let $\Lambda_{N}^{i} \equiv \Lambda \cap V_{N}^{l}, i \in V_{\Delta}$.

Proof of Proposition 3.1. First note that, by Lemma 3.5,

$$
\begin{aligned}
P(X(j) & \left.\geqq b_{N}, j \in V_{N}\right) \\
& \leqq E\left[P\left(X(k) \geqq b_{N}, k \in \Lambda_{N} \mid \mathscr{F}_{L}\right) ; X(j) \geqq b_{N}, j \in V_{N} \cap L \mathbb{Z}^{d}\right] \\
& \leqq E\left[\prod_{k \in \Lambda_{N}} P\left(X(k) \geqq b_{N} \mid \mathscr{F}_{L}\right)^{K_{L}} ; X(j) \geqq b_{N}, j \in V_{N} \cap L \mathbb{Z}^{d}\right] \\
& =E\left[\prod_{k \in \Lambda_{N}}\left(1-\phi\left(-\left(\bar{X}(k)-b_{N}\right) / \sqrt{\mathbf{G}^{\mathbf{L}}}\right)\right)^{K_{L}} ; X(j) \geqq b_{N}, j \in V_{N} \cap L \mathbb{Z}^{d}\right] .
\end{aligned}
$$

For $a>0$, let

$$
\bar{I}_{N}^{i} \equiv\left\{j \in \Lambda_{N}^{i}: \bar{X}(j) \leqq \sqrt{a \log N}+b_{N}\right\}, \quad i \in V_{\Delta}
$$

and, for each $0<\delta<1$, define

$$
F_{N}^{i} \equiv\left\{\left|\bar{I}_{N}^{i}\right| \geqq \delta\left|\Lambda_{N}^{i}\right|\right\}
$$


Note that $\phi(x) \geqq \frac{k}{|x|} e^{-x^{2} / 2}$, for some $k>0$ and $x<-1$. Then, on $\bigcup_{i \in V_{\Delta}} F_{N}^{i}$,

$$
\begin{aligned}
\prod_{k \in V_{N}} & \left(1-\phi\left(-\left(\bar{X}(k)-b_{N}\right) / \sqrt{\mathbf{G}^{\mathbf{L}}}\right)\right)^{K_{L}} \leqq\left(1-\phi\left(-\sqrt{a \log N / \mathbf{G}^{\mathbf{L}}}\right)\right)^{\delta K_{L}\left|\Lambda_{N}^{l}\right|} \\
& \leqq\left(1-\frac{k}{\sqrt{a \log N / \mathbf{G}^{\mathbf{L}}}} \exp \left(-a \log N / 2 \mathbf{G}^{\mathbf{L}}\right)\right)^{\delta K_{L} N^{d} L^{-d} \Delta^{-d}} \\
& \leqq \exp \left(-\frac{k_{1}}{\sqrt{\log N} N^{d-a / 2} \mathbf{G}^{\mathbf{L}}}\right)
\end{aligned}
$$

where $k_{1}=k(\delta, L, \Delta)=\frac{k}{\sqrt{a / \mathbf{G}^{\mathbf{L}}}} \delta K_{L} L^{-d} \Delta^{-d}$.

Choose now $a<2 \alpha \mathbf{G}^{\mathrm{L}}$, then

$$
E\left[\prod_{k \in V_{N}}\left(1-\phi\left(-\left(\bar{X}(k)-b_{N}\right) / \sqrt{\mathbf{G}^{\mathbf{L}}}\right)\right)^{K_{L}} ; \bigcap_{i \in V_{\Delta}} F_{N}^{i}\right] \leqq k_{2} \exp \left(-k_{1} N^{d-\alpha+\delta^{\prime}}\right),
$$

for some $k_{2}, \delta^{\prime}>0$, and can therefore be neglected.

Once we know that, on $\Omega_{N}$, most of the $\bar{X}(j), j \in \Lambda_{N}^{i}$, are at the level $\sqrt{2 \alpha \mathbf{G}^{\mathbf{L}}}$, we can estimate the upper bound with computations related to averages on $\Lambda_{N}^{l}$. This is particularly simple since we are dealing with a Gaussian field. Thus, let

$$
S_{N}^{i} \equiv \frac{1}{\left|\Lambda_{N}^{i}\right|} \sum_{j \in \Lambda_{N}^{l}} X(j), \quad \bar{S}_{N}^{i} \equiv \frac{1}{\left|\Lambda_{N}^{i}\right|} \sum_{j \in \Lambda_{N}^{l}} \bar{X}(j)
$$

Then, on $\left(\bigcup_{i \in V_{\Delta}} F_{N}^{i}\right)^{\mathrm{C}} \cap\left\{X(j) \geqq b_{N}, j \in V_{N} \cap L \mathbb{Z}^{d}\right\}$, for each $i \in V_{\Delta}$,

$$
\begin{aligned}
\overline{S^{l}}{ }_{N}= & \frac{1}{\left|\Lambda_{N}^{l}\right|} \sum_{j \in\left(I_{N}^{i}\right) \mathbf{c}} \bar{X}(j)+\frac{1}{\left|\Lambda_{N}^{i}\right|} \sum_{j \in I_{N}^{i}} \bar{X}(j) \\
= & \frac{1}{\left|\Lambda_{N}^{i}\right|} \sum_{j \in\left(I_{N}^{i}\right) \mathbf{c}} \bar{X}(j)+\frac{1}{\left|\Lambda_{N}^{i}\right|} \sum_{j \in I_{N}^{i}} \sum_{k \in L \mathbb{Z}^{d} \cap V_{N}} q^{L}(j, k) X(k) \\
& +\frac{1}{\left|\Lambda_{N}^{i}\right|} \sum_{j \in I_{N}^{i}} \sum_{k \in L \mathbb{Z}^{d} \backslash V_{N}} q^{L}(j, k) X(k) \\
\geqq & (1-\delta)\left(\sqrt{a \log N}+b_{N}\right)+\delta\left(b_{N} \wedge 0\right)+Z_{N}^{i},
\end{aligned}
$$

where

$$
Z_{N}^{i} \equiv \frac{1}{\left|\Lambda_{N}^{i}\right|} \sum_{j \in I_{N}^{i}} \sum_{k \in L \mathbb{Z}^{d} \backslash V_{N}} q^{L}(j, k) X(k)
$$

In Lemma 3.7 below we show that, for each $\kappa>0$,

$$
\limsup _{N \rightarrow \infty} \frac{1}{N^{d-\alpha} \log N} \log P\left(\bigcup_{i \in V_{\Delta}}\left\{Z_{N}^{i} 1_{\left\{\left|I_{N}^{l}\right|<\delta\left|\Lambda_{N}^{i}\right|\right\}}>\sqrt{\kappa \log N}\right\}\right)=-\infty .
$$


This together with the above yields

$$
\begin{aligned}
& \limsup _{N \rightarrow \infty} \frac{1}{N^{d-\alpha} \log N} \log P\left(X(i) \geqq b_{N}, i \in V_{N}\right) \\
& \leqq \limsup _{N \rightarrow \infty} \frac{1}{N^{d-\alpha} \log N} \log P\left(\bigcap_{i \in V_{\Delta}}\left\{\bar{S}_{N}^{i} \geqq(1-\delta)\left(\sqrt{a \log N}+b_{N}\right)+\delta\left(b_{N} \wedge 0\right)\right\}\right)
\end{aligned}
$$

for any $0<\delta<1$ and $a<2 \alpha \mathbf{G}^{\mathbf{L}}$. Set $a_{N}=(1-\delta)\left(\sqrt{a \log N}+b_{N}\right)+\delta\left(b_{N} \wedge 0\right)$, then

$$
\lim _{N \rightarrow \infty} \frac{a_{N}}{\sqrt{\log N}}=(1-\delta)(\sqrt{a}+b)+\delta(b \wedge 0) .
$$

Since $\left\{\bar{S}_{N}^{i}: i \in V_{\Delta}\right\}$ is centered Gaussian, for each $\left\{f_{i}, i \in V_{\Delta}\right\}$ non-negative

$$
\begin{aligned}
P\left(\bigcap_{i \in V_{\Delta}}\left\{\bar{S}_{N}^{i} \geqq a_{N}\right\}\right) & \leqq P\left(\sum_{i \in V_{\Delta}} f_{i} \bar{S}_{N}^{i} \geqq a_{N} \sum_{i \in V_{\Delta}} f_{i}\right) \\
& \leqq \frac{1}{2} \exp \left(-\frac{a_{N}^{2}\left(\sum_{i \in V_{\Delta}} f_{i}\right)^{2}}{2 \operatorname{var}\left(\sum_{i \in V_{\Delta}} f_{i} \bar{S}_{N}^{i}\right)}\right)
\end{aligned}
$$

Next, by the linearity of the conditional expectation and Jensen's inequality,

$$
\operatorname{var}\left(\sum_{i \in V_{\Delta}} f_{i} \bar{S}_{N}^{i}\right) \leqq \operatorname{var}\left(\sum_{i \in V_{\Delta}} f_{i} S_{N}^{i}\right)
$$

Define now

$$
h(x) \equiv \sum_{i \in V_{\Delta}} f_{i} 1_{\bar{V}_{\Delta}^{l}}(x) \quad \text { with } \quad \bar{V}_{\Delta}^{i}=i / \Delta+V(1 / \Delta) \subseteq V,
$$

where $V(1 / \Delta) \equiv\left[-\frac{1}{2 \Delta}, \frac{1}{2 \Delta}\right]$, then

$$
\sum_{i \in V_{\Delta}} f_{i} S_{N}^{i}=\frac{\Delta^{d}}{\left|\Lambda_{\Delta}\right|} \sum_{j \in \Lambda_{N}} h(j / N) X(j), \quad \sum_{i \in V_{\Delta}} f_{i}=\Delta^{d} \int_{V} h(x) d x
$$

and therefore

$$
\operatorname{var}\left(\sum_{i \in V_{\Delta}} f_{i} S_{N}^{i}\right)=\frac{\Delta^{2 d}}{\left|\Lambda_{N}\right|^{2}} \sum_{i, j \in \Lambda_{N}} h(i / N) h(j / N) G(i, j) .
$$

Thus by Lemma 2.2 of [0] in case $\left(\mathrm{a}^{\prime}\right)$, respectively Lemma A.8 below in case (b),

$$
\lim _{N \rightarrow \infty} \frac{1}{N^{d-\alpha}} \frac{\left(\sum_{i \in V_{\Delta}} f_{i}\right)^{2}}{\operatorname{var}\left(\sum_{i \in V_{\Delta}} f_{i} S_{N}^{i}\right)}=\frac{\left(\int_{V} h(x) d x\right)^{2}}{\int_{V} \int_{V} h(x) h(y) g_{\alpha}(x-y) d x d y} \equiv \mathbf{C}(h) .
$$


As a consequence we get, by (3.6) with $a=2 \alpha \mathbf{G}^{\mathbf{L}}$,

$$
\begin{aligned}
\limsup _{N \rightarrow \infty} \frac{1}{N^{d-\alpha} \log N} \log P(X(i) & \left.\geqq b_{N}, i \in V_{N}\right) \\
& \leqq-\left((1-\delta)\left(\sqrt{2 \alpha \mathbf{G}^{\mathbf{L}}}+b\right)+\delta(b \wedge 0)\right)^{2} \frac{\mathbf{C}(h)}{2} .
\end{aligned}
$$

Taking first $\delta \rightarrow 0$, then $L \rightarrow \infty$ and $\Delta \rightarrow \infty$ yields the constant $(\sqrt{2 \alpha \mathbf{G}}+b)^{2} \frac{\mathbf{C}^{\prime}}{2}$, cf. (3.4), where

$$
\mathbf{C}^{\prime}=\sup \{\mathbf{C}(h): h \text { piecewise constant on a uniform grid }\}=\operatorname{cap}_{\alpha}(V)
$$

by Lemma A.7 in case (b), respectively, Lemma 2.1 of [0] and (A.4), in case (a).

Lemma 3.7. For each $\kappa>0$

$$
\limsup _{N \rightarrow \infty} \frac{1}{N^{d-\alpha} \log N} \log P\left(\bigcup_{i \in V_{\Delta}}\left\{Z_{N}^{i} 1_{\left\{\left|\bar{I}_{N}^{i}\right|<\delta\left|\Lambda_{N}^{i}\right|\right\}}>\sqrt{\kappa \log N}\right\}\right)=-\infty .
$$

Proof. Throughout this proof, $c$ will denote a constant, which depends on $L, \kappa$ but not on $N$, whose value may change from line to line. Let

$$
\mathscr{P}_{i}=P\left(Z_{N}^{i} 1_{\left\{\left|\bar{I}_{N}^{i}\right| \leqq \delta\left|\Lambda_{N}^{i}\right|\right\}} \geqq \sqrt{\kappa \log N}\right) .
$$

Note first that since $\left|V_{\Delta}\right|$ is bounded independently of $N$, it is enough to show that for each $i$,

$$
\limsup _{N \rightarrow \infty} \frac{1}{N^{d-x} \log N} \log \mathscr{P}_{i}=-\infty
$$

We will show that there exists $\varepsilon^{\prime}>0$ such that

$$
\lim _{N \rightarrow \infty} N^{-d-\varepsilon^{\prime}} \log \mathscr{P}_{i}=-\infty
$$

To this end, let $I_{\beta}$ denote a set of $|\beta| \leqq \delta\left|\Lambda_{N}^{i}\right|$ indices in $\Lambda_{N}^{i}$. Note that the number of admissible $\beta$ is bounded by $2^{\left|\Lambda_{N}^{i}\right|}$. Let

$$
Z_{\beta}=\left|\Lambda_{N}^{i}\right|^{-1} \sum_{j \in I_{\beta}} \sum_{k \in L \mathbb{Z}^{d} \backslash V_{N}} q^{L}(j, k) X(k) .
$$

For fixed $I_{\beta}, Z_{\beta}$ is Gaussian, then

$$
\mathscr{P}_{i} \leqq 2^{\left|\Lambda_{N}^{i}\right|} \max _{\beta} P\left(Z_{\beta}>\sqrt{\kappa \log N}\right) \leqq 2^{\left|\Lambda_{N}^{i}\right|} \frac{1}{2} \exp \left(-\frac{\kappa \log N}{2 \max _{\beta} E\left[Z_{\beta}^{2}\right]}\right)
$$

The lemma thus follows if we can show that there exists an $\varepsilon>0$ such that

$$
\max _{\beta} E\left[Z_{\beta}^{2}\right] \leqq c N^{-d-\varepsilon}
$$


We show (3.8) in case $\left(b^{\prime}\right)$, the proof in case $\left(a^{\prime}\right)$ is similar. To see (3.8), note that, by (A.13) below, for all $\alpha^{\prime}<\alpha$,

$$
\begin{aligned}
& E\left[Z_{\beta}^{2}\right] \leqq c N^{-2 d} \sum_{j, j^{\prime} \in I_{\beta}} \sum_{k, k^{\prime} \in L \mathbb{Z}^{d} \backslash V_{N}} q^{L}(j, k) q^{L}\left(j^{\prime}, k^{\prime}\right) G\left(k, k^{\prime}\right) \\
& \leqq c N^{-2 d} \sum_{j, j^{\prime} \in \Lambda_{N}} \sum_{k, k^{\prime} \in L \mathbb{Z}^{d} \backslash V_{N}, k \neq k^{\prime}}|j-k|^{-d-\alpha^{\prime}}\left|j^{\prime}-k^{\prime}\right|^{-d-\alpha^{\prime}}\left|k-k^{\prime}\right|^{-d+\alpha} \\
& =c N^{-d-2 \alpha^{\prime}+\alpha} \sum_{j, j^{\prime} \in \Lambda_{N}} \sum_{k, k^{\prime} \in L \mathbb{Z}^{d} \backslash V_{N}, k \neq k^{\prime}}\left|\frac{j}{N}-\frac{k}{N}\right|^{-d-\alpha^{\prime}}\left|\frac{j^{\prime}}{N}-\frac{k^{\prime}}{N}\right|^{-d-x^{\prime}} \\
& \times\left|\frac{k}{N}-\frac{k^{\prime}}{N}\right|^{-d+\alpha} \frac{1}{\left|\Lambda_{N}\right|^{4}} \text {. }
\end{aligned}
$$

By Riemannn integration

$$
\begin{array}{r}
\lim _{N \rightarrow \infty} \sum_{j, j^{\prime} \in \Lambda_{N}} \sum_{k, k^{\prime} \in L \mathbb{Z}^{d} \backslash V_{N}, k \neq k^{\prime}}\left|\frac{j}{N}-\frac{k}{N}\right|^{-d-x^{\prime}}\left|\frac{j^{\prime}}{N}-\frac{k^{\prime}}{N}\right|^{-d-x^{\prime}}\left|\frac{k}{N}-\frac{k^{\prime}}{N}\right|^{-d+\alpha} \frac{1}{\left|\Lambda_{N}\right|^{4}} \\
=\iint_{V} \int_{V^{\mathbf{C}}} \int_{V}\left|x_{1}-x_{2}\right|^{-d-\alpha^{\prime}}\left|x_{2}-x_{3}\right|^{-d+\alpha}\left|x_{3}-x_{4}\right|^{-d-\alpha^{\prime}} d x_{1} d x_{2} d x_{3} d x_{4}<\infty .
\end{array}
$$

In order to verify that the last integral is finite, we may replace $V=[1 / 2,1 / 2]^{d}$ by the unit ball $B=\left\{x \in \mathbb{R}^{d}:|x| \leqq 1\right\}$ and use the inequality

$$
\int_{B}\left|x_{1}-x_{2}\right|^{-d-\alpha^{\prime}} d x_{1} \leqq \begin{cases}c|| x_{2}|-1|^{-\alpha^{\prime}} & 1<\left|x_{2}\right| \leqq 3 \\ c\left|x_{2}\right|^{-d-\alpha^{\prime}} & 3 \leqq\left|x_{2}\right|<\infty\end{cases}
$$

Choosing now $\alpha-\alpha^{\prime}$ small enough and using (3.9), the lemma follows.

We conclude this section with the proof of Proposition 1.7:

Proof of Proposition 1.7. We begin with a Gibbsian description of $P^{\varepsilon}$ : Let $P_{k}^{\varepsilon}\left(\cdot \mid \mathscr{F}_{\{k\}} \mathbf{C}\right)$ be the conditional law of $X(k)$ given by $\mathscr{F}_{\{k\}} \mathbf{C}$. Then

$$
P_{k}^{\varepsilon}\left(\cdot \mid \mathscr{F}_{\{k\}} \mathbf{C}\right)=\mathscr{N}((1-\varepsilon) \tilde{X}(k) ; 1)
$$

where $\tilde{X}(k)=\sum_{j \neq k} Q(k, j) X(j)$. We can view $P^{\varepsilon}$ as the unique Gibbs state to the interaction potential $\mathscr{U}=\left\{U_{F}: \emptyset \neq F \subset \subset \mathbb{Z}^{d}\right\}$

$$
U_{F}(X)= \begin{cases}\frac{X(k)^{2}}{2} & F=\{k\} \\ -(1-\varepsilon) Q(k, j) X(k) X(j) & F=\{k, j\} \\ 0 & |F|>2\end{cases}
$$


with reference measure the Lebesgue measure $d x$ on $\mathbb{R}$. Consider now the new interaction potential $\mathscr{U}^{+}=\left\{U_{F}^{+}: \emptyset \neq F \subset \subset \mathbb{Z}^{d}\right\}$

$$
U_{F}^{+}(X)= \begin{cases}\frac{X(k)^{2}}{2}+\infty 1_{\{X(k)<0\}} & F=\{k\} \\ -(1-\varepsilon) Q(k, j) X(k) X(j) & F=\{k, j\} \\ 0 & |F|>2 .\end{cases}
$$

Let $\mathscr{G}_{0}\left(\mathscr{U}^{+}\right)$be the associated set of translation invariant tempered Gibbs states on $\Omega^{+} \equiv\left(\mathbb{R}^{+}\right)^{\mathbb{Z}^{d}} . \mathscr{G}_{0}\left(\mathscr{U}^{+}\right) \neq \emptyset$ since $\mathscr{U}^{+}$is superregular and superstable, cf. Definition 1.7 and Example 1.12 of [9]. We claim that $\mathscr{G}_{0}\left(\mathscr{U}^{+}\right)$consists of a unique point $\left\{Q^{*}\right\}$. This follows from Dobrushin's uniqueness criterion: let

$$
P_{k}^{\varepsilon,+}\left(d x \mid \mathscr{F}_{\{k\}} \mathbf{C}\right)=v^{+}(d x \mid \tilde{X}(k))=\frac{\exp \left(-x^{2} / 2+(1-\varepsilon) \tilde{X}(k) x\right)}{\int_{0}^{\infty} \exp \left(-y^{2} / 2+(1-\varepsilon) \tilde{X}(k) y\right) d y} 1_{\{x \geqq 0\}} d x
$$

be the conditional law of $X(k)$ given $\mathscr{F}_{\{k\}} \mathrm{C}$ for any Gibbs' state in $\mathscr{G}_{0}\left(\mathscr{U}^{+}\right)$. Next let $W: \mathscr{M}_{1}(\mathbb{R}) \times \mathscr{M}_{1}(\mathbb{R}) \longrightarrow[0, \infty]$ be the Wasserstein metric with respect to the Euclidean norm $|\cdot|$ on $\mathbb{R}$, that is

$$
\begin{aligned}
W(v, \mu) & =\sup \left\{\int_{\mathbb{R}^{+}} f(x) v(d x)-\int_{\mathbb{R}^{+}} f(x) \mu(d x): f \in C\left(\mathbb{R}^{+}\right),\right. \\
\delta(f) & \left.=\sup _{x \neq y} \frac{|f(x)-f(y)|}{|x-y|} \leqq 1\right\} .
\end{aligned}
$$

Set

$D(k, j) \equiv \sup \left\{\frac{W\left(v^{+}(\cdot \mid \tilde{X}(k)), v^{+}(\cdot \mid \tilde{Y}(k))\right)}{|X(j)-Y(j)|}: X, Y \in \Omega^{+}\right.$with $\left.X(i)=Y(i), i \neq j\right\}$.

Then, if $v^{+}(f \mid y)=\int_{\mathbb{R}^{+}} f(z) v^{+}(d z \mid y)$, respectively $v^{+}(x \mid y)=\int_{\mathbb{R}^{+}} z v^{+}(d z \mid y)$, we get by the Cauchy Schwarz inequality

$$
\begin{aligned}
\left|\frac{d}{d y} v^{+}(f \mid y)\right|= & (1-\varepsilon)\left|\int_{\mathbb{R}^{+}}\left(f(z)-v^{+}(f \mid y)\right)\left(z-v^{+}(x \mid y)\right) v^{+}(d z \mid y)\right| \\
\leqq & (1-\varepsilon)\left(\int_{\mathbb{R}^{+}}\left(f(z)-v^{+}(f \mid y)\right)^{2} v^{+}(d z \mid y)\right)^{1 / 2} \\
& \times\left(\int_{\mathbb{R}^{+}}\left(z-v^{+}(x \mid y)\right)^{2} v^{+}(d z \mid y)\right)^{1 / 2} \\
\leqq & (1-\varepsilon) \delta(f) \operatorname{var}\left(v^{+}(\cdot \mid y)\right) .
\end{aligned}
$$

This yields

$$
D(k, j) \leqq(1-\varepsilon) Q(k, j) \sup _{y \geqq 0} \operatorname{var}\left(v^{+}(\cdot \mid y)\right),
$$

with

$$
\operatorname{var}\left(v^{+}(\cdot \mid y)\right)=\inf _{b \geqq 0} \frac{\int_{0}^{\infty}(x-b)^{2} e^{-(x-y)^{2} / 2} d x}{\int_{0}^{\infty} e^{-(x-y)^{2} / 2} d x} \leqq \frac{\int_{0}^{\infty}(x-y)^{2} e^{-(x-y)^{2} / 2} d x}{\int_{0}^{\infty} e^{-(x-y)^{2} / 2} d x} \leqq 1 .
$$


Thus

$$
\sup _{k \in \mathbb{Z}^{d}} \sum_{j \neq k} D(k, j) \leqq(1-\varepsilon)<1,
$$

and we have uniqueness by Dobrushin's criterion, cf. Theorem 4 in [5]. Using the standard variational principle, one then verifies that $Q^{*}$ minimizes the specific entropy $\mathbf{h}\left(\cdot \mid P^{\varepsilon}\right)$ under the constraint $\Pi(Q) \in \mathscr{A}$, cf. [9]. Also the upper bound large deviations yields

$$
\limsup _{N \rightarrow \infty} \frac{1}{\left|V_{N}\right|} \log P^{\varepsilon}\left(\Omega_{N}\right) \leqq-\inf _{Q: \Pi(Q) \in \mathscr{A}} \mathbf{h}\left(Q \mid P^{\varepsilon}\right)=-\mathbf{h}\left(Q^{*} \mid P^{\varepsilon}\right) .
$$

Hypercontractivity shows that $\mathbf{h}\left(Q \mid P^{\varepsilon}\right) \geqq c \mathbf{H}_{1}\left(\prod(Q) \mid \prod\left(P^{\varepsilon}\right)\right)$ for some constant $c>0$, cf. Sect. 5.4 [3], and hence $\mathbf{h}\left(Q^{*} \mid P^{\varepsilon}\right)>0$. We cannot apply directly the large deviation principle for the lower bound, since $\mathscr{A}$ has a void interior. However $Q^{*}\left(\Omega_{N}\right)=1$ and this implies

$$
\liminf _{N \rightarrow \infty} \frac{1}{\left|V_{N}\right|} \log P^{\varepsilon}\left(\Omega_{N}\right) \geqq-\mathbf{h}\left(Q^{*} \mid P^{\varepsilon}\right),
$$

cf. Proof of Lemma 2.3. Finally, the convergence of $P^{\varepsilon}\left(\cdot \mid \Omega_{N}\right)$ follows along the same pattern as the proof of Theorem 3.5 in [4].

\section{Entropic Repulsion}

In this section we give a proof of Proposition 1.3. Our main technique is the monotonicity or FKG property of the measure $P$. Our starting point is Proposition 4.1 below. Note that only (4.2) will be actually needed in the proof of Proposition 1.3.

Proposition 4.1. Let $a<2 \alpha \mathbf{G}, b>2 \alpha \mathbf{G}$ and $\delta \in(0,1)$, then

$$
\lim _{N \rightarrow \infty} P\left(L_{V_{N}}[0, \sqrt{a \log N}] \geqq \delta \mid \Omega_{N}\right)=0,
$$

and

$$
\lim _{N \rightarrow \infty} P\left(L_{V_{N}}[\sqrt{b \log N}, \infty) \geqq \delta \mid \Omega_{N}\right)=0
$$

For fixed $L \in 2 \mathbb{N}^{+}$, define $\mathscr{F}_{L}, \Lambda_{N}, \bar{X}(i), G^{L}(i, j), i, j \in \Lambda_{N}$ as in Sect. 3 and set

$$
L_{\Lambda_{N}}=\frac{1}{\left|\Lambda_{N}\right|} \sum_{k \in \Lambda_{N}} \delta_{X(k)} \text {. }
$$

The crucial step in the proof of (4.2) is the following.

Lemma 4.4. Let $a<2 \alpha \mathbf{G}^{\mathbf{L}}$, then for each $\delta>0$,

$$
\lim _{N \rightarrow \infty} P\left(L_{\Lambda_{N}}[0, \sqrt{a \log N}] \geqq \delta \mid \Omega_{N}\right)=0 .
$$




\section{Proof. Set}

$$
\begin{aligned}
& I_{N}(a)=\left\{j \in \Lambda_{N}: X(j) \leqq \sqrt{a \log N}\right\}, \\
& \bar{I}_{N}(a)=\left\{j \in \Lambda_{N}: \bar{X}(j) \leqq \sqrt{a \log N}\right\}, \bar{I}_{N}(a)^{\mathrm{C}}=\left\{j \in \Lambda_{N}: \bar{X}(j)>\sqrt{a \log N}\right\} .
\end{aligned}
$$

Following the argument of the proof of Proposition 3.1, we know that for each $\delta>\delta^{\prime}>0$ and $a<a^{\prime}<2 \alpha \mathbf{G}^{\mathbf{L}}$

$$
P\left(\left|\bar{I}_{N}\left(a^{\prime}\right)\right| \geqq \delta^{\prime}\left|\Lambda_{N}\right| ; \Omega_{N}\right) \leqq \exp \left(-c N^{d-\alpha+\varepsilon}\right)
$$

for some $c, \varepsilon>0$. By (2.2), this implies

$$
\lim _{N \rightarrow \infty} P\left(\left|\bar{I}_{N}\left(a^{\prime}\right)\right| \geqq \delta^{\prime}\left|\Lambda_{N}\right| \mid \Omega_{N}\right)=0 .
$$

Since

$$
\begin{aligned}
\left\{L_{\Lambda_{N}}[0, \sqrt{a \log N}] \geqq \delta\right\} & =\left\{\left|I_{N}(a)\right| \geqq \delta\left|\Lambda_{N}\right|\right\} \\
& =\left\{\left|I_{N}(a)\right| \geqq \delta\left|\Lambda_{N}\right|,\left|\bar{I}_{N}\left(a^{\prime}\right)\right|<\delta^{\prime}\left|\Lambda_{N}\right|\right\} \cup\left\{\left|I_{N}(a)\right|\right. \\
& \left.\geqq \delta\left|\Lambda_{N}\right|,\left|\bar{I}_{N}\left(a^{\prime}\right)\right| \geqq \delta^{\prime}\left|\Lambda_{N}\right|\right\} \\
& \subseteq\left\{\left|I_{N}(a) \cap \bar{I}_{N}\left(a^{\prime}\right)^{\mathbf{C}}\right| \geqq\left(\delta-\delta^{\prime}\right)\left|\Lambda_{N}\right|\right\} \cup\left\{\left|\bar{I}_{N}\left(a^{\prime}\right)\right|\right. \\
& \left.\geqq \delta^{\prime}\left|\Lambda_{N}\right|\right\},
\end{aligned}
$$

all we have to show is

$$
\lim _{N \rightarrow \infty} P\left(\left|I_{N}(a) \cap \bar{I}_{N}\left(a^{\prime}\right)^{\mathbf{C}}\right| \geqq\left(\delta-\delta^{\prime}\right)\left|\Lambda_{N}\right| \mid \Omega_{N}\right)=0 .
$$

Let $k \in I_{N}(a) \cap \bar{I}_{N}\left(a^{\prime}\right)^{C}$ then

$$
\bar{X}(k)-X(k) \geqq\left(\sqrt{a^{\prime}}-\sqrt{a}\right) \sqrt{\log N} .
$$

Thus on $\left\{\left|I_{N}(a) \cap \bar{I}_{N}\left(a^{\prime}\right)^{\mathbf{C}}\right| \geqq\left(\delta-\delta^{\prime}\right)\left|\Lambda_{N}\right|\right\}$, we have

$$
\frac{1}{\left|\Lambda_{N}\right|} \sum_{k \in \Lambda_{N}}|X(k)-\bar{X}(k)| \geqq\left(\delta-\delta^{\prime}\right)\left(\sqrt{a^{\prime}}-\sqrt{a}\right) \sqrt{\log N} .
$$

Note that $G^{L}(k, j)=\operatorname{cov}\left(X(k), X(j) \mid \mathscr{F}_{L}\right)=E[(X(k)-\bar{X}(k))(X(j)-\bar{X}(j))]$ is rapidly decaying, cf. (3.2), but this implies

$$
\begin{gathered}
\limsup _{N \rightarrow \infty} \frac{1}{N^{d} \log N} \log P\left(\frac{1}{\left|\Lambda_{N}\right|} \sum_{k \in \Lambda_{N}}|X(k)-\bar{X}(k)|\right. \\
\left.\geqq\left(\delta-\delta^{\prime}\right)\left(\sqrt{a^{\prime}}-\sqrt{a}\right) \sqrt{\log N}\right)<-c
\end{gathered}
$$

for some $c>0$ depending on $L, \delta-\delta^{\prime}$ and $\sqrt{a^{\prime}}-\sqrt{a}$. This together with (2.2) proves (4.5) and concludes the proof.

Proof of (4.2). For $\ell \in V_{L}$, define $\mathscr{F}_{L}(\ell)=\sigma\left(X(k+\ell), k \in L \mathbb{Z}^{d}\right), \Lambda(\ell)=(L / 2+$ $\ell, \ldots, L / 2+\ell)+L \mathbb{Z}^{a}, \Lambda_{N}(\ell)=\Lambda(\ell) \cap V_{N}$ and

$$
I_{N}(a, \ell)=\left\{j \in \Lambda_{N}(\ell): X(j) \leqq \sqrt{a \log N}\right\} .
$$


Using the argument of the preceding lemma, one shows that for each $\ell \in V_{L}, a<$ $2 \alpha \mathbf{G}^{\mathbf{L}}$ and $\delta>0$

$$
\lim _{N \rightarrow \infty} P\left(\left|I_{N}(a, \ell)\right| \geqq \delta\left|\Lambda_{N}(\ell)\right| \mid \Omega_{N}\right)=0
$$

Also

$$
\begin{aligned}
\left\{L_{V_{N}}[0, \sqrt{a \log N}] \geqq \delta\right\} & =\left\{\left|\left\{k \in V_{N}: 0 \leqq X(k) \leqq \sqrt{a \log N}\right\}\right| \geqq \delta\left|V_{N}\right|\right\} \\
& \subseteq \bigcup_{\ell \in V_{L}}\left\{\left|I_{N}(a, \ell)\right| \geqq \delta \Lambda_{N}(\ell)\right\} .
\end{aligned}
$$

This implies (4.2) for each $a<2 \alpha \mathbf{G}^{\mathbf{L}}$, and the result follows with $L \rightarrow \infty$ by (3.4).

Proof of (1.4). Let us recall (1.4):

$$
\lim _{N \rightarrow \infty} \sup _{k \in V_{N, \varepsilon}} P\left(X(k) \leqq \sqrt{a \log N} \mid \Omega_{N}\right)=0 .
$$

Consider a small cube, $V_{N}(k)$ centered at $k$ with side length $\leqq(\varepsilon / 3) N$. Let $W_{N}(\ell)$ be a cube centered at $\ell$ with size length $(2 \varepsilon / 3) N$. Then, for $k \in V_{N, \varepsilon}$,

$$
V_{N}(k) \subseteq \bigcap_{\ell \in V_{N}(k)} W_{N}(\ell), \quad \bigcup_{\ell \in V_{N}(k)} W_{N}(\ell) \subseteq V_{N},
$$

and by the FKG property, for each $\ell \in V_{N}(k)$ :

$$
\begin{aligned}
P\left(X(k) \leqq \sqrt{a \log N} \mid \Omega_{N}\right) & \leqq P\left(X(k) \leqq \sqrt{a \log N} \mid X(j) \geqq 0, j \in W_{N}(k)\right) \\
& =P\left(X(k+\ell) \leqq \sqrt{a \log N} \mid X(j) \geqq 0, j \in W_{N}(k+\ell)\right) \\
& \leqq P\left(X(k+\ell) \leqq \sqrt{a \log N} \mid X(j) \geqq 0, j \in V_{N}(k)\right) .
\end{aligned}
$$

Thus, for any $\delta>0$,

$$
\begin{aligned}
P(X(k) \leqq & \left.\sqrt{a \log N} \mid X(j) \geqq 0, j \in V_{N}\right) \leqq \frac{1}{\left|V_{N}(k)\right|} \sum_{\ell \in V_{N}(k)} P(X(k+\ell) \\
& \left.\leqq \sqrt{a \log N} \mid X(j) \geqq 0, j \in V_{N}(k)\right) \\
= & E\left[L_{V_{N}(k)}[0, \sqrt{a \log N}] \mid X(j) \geqq 0, j \in V_{N}(k)\right] \\
\leqq & \leqq P\left(L_{V_{N}(k)}[0, \sqrt{a \log N}]>\delta \mid X(j) \geqq 0, j \in V_{N}(k)\right)
\end{aligned}
$$

Using (4.2), we have

$$
\begin{aligned}
\lim _{N \rightarrow \infty} P & \left(L_{V_{N}(k)}[0, \sqrt{a \log N}]>\delta \mid X(j) \geqq 0, j \in V_{N}(k)\right) \\
& =\lim _{N \rightarrow \infty} P\left(L_{V_{[(\varepsilon / 3) N]}}[0, \sqrt{a \log N}]>\delta \mid X(j) \geqq 0, \quad j \in V_{[(\varepsilon / 3) N]}\right) \\
& =\lim _{N \rightarrow \infty} P\left(L_{V_{N}}[0, \sqrt{a \log ((3 / \varepsilon) N)}]>\delta \mid \Omega_{N}\right)=0 .
\end{aligned}
$$

Since $\delta>0$ is arbitrary, we have the result. 
Remark. 4.6. One could wonder, what may happen with $k$ closer to the boundary of $V_{N}$. For $1>\varepsilon>0$, let $\partial_{\varepsilon} V \equiv \bigcup_{j=1}^{d}\left\{x=\left(x_{1}, \ldots, x_{d}\right) \in \mathbb{R}^{d}:\left|x_{j}\right|=1 / 2,\left|x_{k}\right| \leqq\right.$ $(1-\varepsilon) / 2, k \neq j\}$ and set $\partial V_{N, \varepsilon}=\left\{i \in \mathbb{Z}^{d}: i / N \in \partial_{\varepsilon} V\right\}$, the "interior" of the boundary of $V_{N}$. An adaptation of the above argument shows, for any $1<\alpha \leqq$ $2 \wedge d$ and $a<2(\alpha-1) \mathbf{G}$,

$$
\lim _{N \rightarrow \infty} \sup _{k \in \partial V_{N, \varepsilon}} P\left(X(k) \leqq \sqrt{a \log N} \mid \Omega_{N}\right)=0 .
$$

Next, we are going to show first (1.5), and then (4.3). Our first step in the proof of (1.5) is the following.

\section{Lemma 4.7.}

$$
\limsup _{N \rightarrow \infty} \sup _{k \in V_{N}} \frac{E\left[X(k) \mid \Omega_{N}\right]}{\sqrt{\log N}} \leqq \sqrt{2 \alpha \mathbf{G}} .
$$

Proof. Again, we use FKG: for fixed $m \in \mathbb{N}$, and any $\ell \in V_{m N}, k \in V_{N}$,

$$
\begin{aligned}
E\left[X(k) \mid \Omega_{N}\right] & =E\left[X(k+\ell) \mid X(j+\ell) \geqq 0, j \in V_{N}\right] \\
& \leqq E\left[X(k+\ell) \mid \Omega_{(m+1) N}\right],
\end{aligned}
$$

since $V_{(m+1) N}=\bigcup_{\ell \in V_{m N}}\left(V_{N}+\ell\right)$ and $V_{m N}+k \subset V_{(m+1) N}$. Next for any $h \geqq 0$ with support in $V_{m N}$ and $\beta>0$, we have

$$
\beta\left\langle 1_{V_{m N}}, h\right\rangle_{V_{m N}} E\left[X(k) \mid \Omega_{N}\right] \leqq \beta \sum_{\ell \in V_{m N}} h(\ell) E\left[X(k+\ell) \mid \Omega_{(m+1) N}\right]=E\left[F_{N} \mid \Omega_{(m+1) N}\right],
$$

where $F_{N} \equiv \beta \sum_{\ell \in V_{m N}} h(\ell) X(k+\ell)$. Using the entropy bound

$$
\begin{aligned}
E\left[F_{N} \mid \Omega_{(m+1) N}\right] & \leqq \mathbf{H}_{(m+1) N}\left(P\left(\cdot \mid \Omega_{(m+1) N}\right) \mid P\right)+\log E\left[\exp \left(F_{N}\right)\right] \\
& =-\log P\left(\Omega_{(m+1) N}\right)+\frac{\beta^{2}}{2}\langle h, G h\rangle_{V_{m N}}
\end{aligned}
$$

we get, by taking the best $\beta$,

$$
E\left[X(k) \mid \Omega_{N}\right] \leqq \sqrt{-2 \log P\left(\Omega_{(m+1) N}\right) \frac{\langle h, G h\rangle_{V_{m N}}}{\langle 1, h\rangle_{V_{m N}}^{2}}} .
$$

Further, note that

$$
\begin{aligned}
\sup _{h \geqq 0} \frac{\left\langle 1_{V_{m N}}, h\right\rangle_{V_{m N}}^{2}}{\langle h, G h\rangle_{V_{m N}}} & =\left\langle 1_{V_{m N}}, G_{V_{m N}}^{-1}, 1_{V_{m N}}\right\rangle_{V_{m N}}=\operatorname{cap}_{m N}\left(V_{m N}\right) \\
& =m^{d-\alpha} N^{d-\alpha}\left(\operatorname{cap}_{\alpha}(V)+o(1)\right),
\end{aligned}
$$

and by Theorem 1.1

$$
-\log P\left(\Omega_{(m+1) N}\right)=(m+1)^{d-\alpha} N^{d-\alpha} \log N\left(\alpha \mathbf{G c a p}_{\alpha}(V)+o(1)\right),
$$

and therefore

$$
\limsup _{N \rightarrow \infty} \sup _{k \in V_{N}} \frac{E\left[X(k) \mid \Omega_{N}\right]}{\sqrt{\log N}} \leqq \sqrt{2 \alpha \mathbf{G}(1+1 / m)^{d-\alpha}} .
$$

Now (4.8) follows with $m \rightarrow \infty$. 
Proof of (1.5). We want to show that

$$
\limsup _{N \rightarrow \infty} \sup _{k \in V_{N, \varepsilon}} P\left(X(k) \geqq \sqrt{b \log N} \mid \Omega_{N}\right)=0
$$

for all $b>2 \alpha \mathbf{G}$. Choose $0<\delta<\sqrt{2 \alpha \mathbf{G}}$ and set $\gamma=\sqrt{2 \alpha \mathbf{G}}-\delta$, then, writing $\hat{X}(k)=\frac{X(k)}{\sqrt{\log N}}$, we have

$$
\begin{aligned}
E\left[\hat{X}(k) \mid \Omega_{N}\right] \geqq & \gamma P\left(\gamma \leqq \hat{X}(k)<\sqrt{b} \mid \Omega_{N}\right)+\sqrt{b} P\left(\hat{X}(k) \geqq \sqrt{b} \mid \Omega_{N}\right) \\
= & \gamma\left(1-P\left(\hat{X}(k) \geqq \sqrt{b} \mid \Omega_{N}\right)-P\left(\hat{X}(k) \leqq \gamma \mid \Omega_{N}\right)\right) \\
& +\sqrt{b} P\left(\hat{X}(k) \geqq \beta \mid \Omega_{N}\right) .
\end{aligned}
$$

Thus

$$
P\left(\hat{X}(k) \geqq \sqrt{b} \mid \Omega_{N}\right) \leqq \frac{E\left[\hat{X}(k) \mid \Omega_{N}\right]-\gamma+\gamma P\left(\hat{X}(k) \leqq \gamma \mid \Omega_{N}\right)}{\sqrt{b}-\gamma} .
$$

Since, by (4.8),

$$
\limsup _{N \rightarrow \infty} \sup _{k \in V_{N}} E\left[\hat{X}(k) \mid \Omega_{N}\right] \leqq \sqrt{2 \alpha \mathbf{G}}
$$

and, by (1.4),

$$
\lim _{N \rightarrow \infty} \sup _{k \in V_{N, \varepsilon}} P\left(\hat{X}(k) \leqq \gamma \mid \Omega_{N}\right)=0
$$

we get

$$
\limsup _{N \rightarrow \infty} \sup _{k \in V_{N, \varepsilon}} P\left(X(k) \geqq \sqrt{b \log N} \mid \Omega_{N}\right) \leqq \frac{\delta}{\sqrt{b}-\sqrt{2 \alpha \mathbf{G}}},
$$

which yields the result with $\delta \searrow 0$.

Proof of (4.3). Let $b>\sqrt{2 \alpha \mathbf{G}}$ and $0<\delta<1$, then for any $\varepsilon>0$,

$$
\begin{aligned}
\delta P\left(L_{V_{N}}[\sqrt{b \log N}, \infty) \geqq \delta \mid \Omega_{N}\right) & \leqq E\left[L_{V_{N}}[\sqrt{b \log N}, \infty) \mid \Omega_{N}\right] \\
& =\frac{1}{\left|V_{N}\right|} \sum_{k \in V_{N}} P\left(X(k) \geqq \sqrt{b \log N \mid} \Omega_{N}\right) \\
& \leqq \sup _{k \in V_{N, \varepsilon}} P\left(X(k) \geqq \sqrt{b \log N} \mid \Omega_{N}\right)+c_{d} \varepsilon^{d}
\end{aligned}
$$

for some constant $c_{d}>0$. Thus by (1.5)

$$
\limsup _{N \rightarrow \infty} P\left(L_{V_{N}}[\sqrt{b \log N}, \infty) \geqq \delta \mid \Omega_{N}\right) \leqq \frac{c_{d} \varepsilon^{d}}{\delta}
$$

and (4.3) follows with $\varepsilon \searrow 0$.

\section{Appendix}

In this appendix we show the convergence of the capacity and derive the estimates of the conditional covariances for the $\alpha$-stable case. 
Let $\alpha \in(0, d \wedge 2)$ and let $q_{\alpha}$ be the density of the isotropic symmetric $\alpha$-stable law on $\mathbb{R}^{d}$ with characteristic function given by

$$
\int_{\mathbb{R}^{d}} e^{i t \cdot x} q_{\alpha}(x) d x=e^{-\rho|t|^{\alpha}}, \quad t \in \mathbb{R}^{d},
$$

for some $\rho>0$. Define $Q$ as in the introduction and let $Q^{n}$ be the $n^{\text {th }}$ product of $Q$.

Lemma A.2. Assume (b) or $\left(\mathrm{b}^{\prime}\right)$, then

$$
\lim _{|k| \rightarrow \infty}|k|^{d+\alpha} Q(k, 0)=c_{\alpha}
$$

and

$$
\sup _{j, k \in \mathbb{Z}^{d}} Q^{n}(j, k) \leqq c n^{-d / \alpha}
$$

for some $c, c_{\alpha}>0$. Also

$$
\lim _{|k| \rightarrow \infty}|k|^{d-\alpha} G(k, 0)=\omega_{\alpha, d} \equiv \frac{\int_{\mathbb{R}^{d}} \psi(x)|x|^{-\alpha} d x}{(2 \pi)^{d} c_{\alpha} \int_{\mathbb{R}^{d}}(1-\psi(x))|x|^{-1-\alpha} d x},
$$

with $\psi(x)=\frac{1}{d} \sum_{i=1}^{d} \cos x_{i}$.

Proof. The first equality, (A.3), follows from the definition of $Q$ and (A.1). As for the proof of (A.4) and (A.5), we use harmonic analysis as in Sect. 7, Sect. 8 of [13], cf. in particular the proof of P6, see also [12], Propositions 2.3, 2.4 and 5.2: Let

$$
\hat{Q}(\theta)=\sum_{k \in \mathbb{Z}^{d}} Q(0, k) e^{i k \cdot \theta}, \quad \theta \in(-\pi, \pi]^{d},
$$

be the Fourier transform of $Q$, then

$$
\lim _{|\theta| \rightarrow 0}|\theta|^{-\alpha}(1-\hat{Q}(\theta))=\gamma_{\alpha, d} \equiv c_{\alpha} \int_{\mathbb{R}^{d}}(1-\psi(x))|x|^{-1-\alpha} d x,
$$

cf. Example 2 Sect. 8 of [13]. Note that $\Psi(\theta)=|\hat{Q}(\theta)|^{2}$ is the Fourier transform of $Q^{2}$. Since $Q$ is strongly aperiodic by assumption, $\Psi(\theta)=1$ if and only if $\theta \in$ $(2 \pi) \mathbb{Z}^{d}$, cf. P8 of Sect. 7 of [13]. Also the above shows

$$
\lim _{|\theta| \rightarrow 0}|\theta|^{-\alpha}(1-\Psi(\theta))=\lim _{|\theta| \rightarrow 0}|\theta|^{-\alpha}(1-\hat{Q}(\theta))(1+\hat{Q}(\theta))=2 \gamma_{\alpha, d} .
$$

Thus there exists $\lambda>0$, such that

$$
0 \leqq \Psi(\theta) \leqq 1-\lambda|\theta|^{\alpha} \leqq e^{-\lambda|\theta|^{\alpha}}, \quad \theta \in(-\pi, \pi]^{d} .
$$

But

$$
(2 \pi)^{d} Q^{2 n}(j, k)=\int_{(-\pi, \pi]^{d}} e^{-i(j-k) \theta} \Phi(\theta)^{n} d \theta \leqq \int_{(-\pi, \pi]^{d}} e^{-\lambda \cdot n|\theta|^{\alpha}} d \theta \leqq c n^{-d / \alpha},
$$

and the same bound holds if $Q^{2 n}(j, k)$ is replaced by $Q^{2 n+1}(j, k)$. This proves (A.4). Next note that, if $\hat{G}$ denotes the Fourier transform of $G$, then $\hat{G}(\theta)=(1-\hat{Q}(\theta))^{-1}$ with

$$
\lim _{\theta \rightarrow 0}|\theta|^{\alpha} \hat{G}(\theta)=\frac{1}{\gamma_{\alpha, d}}
$$


and

$$
G(k, 0)=\frac{1}{(2 \pi)^{d}} \int_{(-\pi, \pi]^{d}} \hat{G}(\theta) e^{-i k \theta} d \theta
$$

This yields

$$
\lim _{|k| \rightarrow \infty}|k|^{d-\alpha} G(k, 0)=\frac{1}{(2 \pi)^{d} \gamma_{\alpha, d}} \int_{\mathbb{R}^{d}} \psi(x)|x|^{-x} d x=\omega_{\alpha, d} .
$$

Let $g_{\alpha}(x)=\omega_{\alpha, d}|x|^{-d+\alpha}$ be the Riesz kernel and define the integral operators $K$ and $K_{V}$ on $L^{2}\left(\mathbb{R}^{d}\right)$ and $L^{2}(V)$,

$$
K \phi(x) \equiv \int_{\mathbb{R}^{d}} g_{\alpha}(x-y) \phi(y) d y, \quad K_{V} \phi(x) \equiv \int_{V} g_{\alpha}(x-y) \phi(y) d y .
$$

$K_{V}$ is a positive definite, compact self-adjoint operator with $\left(L^{2}(V)\right.$-normalized) eigenfunctions $\left\{e_{n}\right\}$ and eigenvalues $\left\{\lambda_{1}(V)>\lambda_{2}(V) \geqq \cdots\right\}$. For $\phi \in C^{2}\left(\mathbb{R}^{d}\right) \cap$ $L^{2}\left(\mathbb{R}^{d}\right)$ let

$$
K^{-1} \phi(x) \equiv \int_{\mathbb{R}^{d}}(\phi(y)-\phi(x)) g_{\alpha}^{-1}(x-y) d y,
$$

where

$$
g_{\alpha}^{-1}(x) \equiv c_{\alpha}|x|^{-d-\alpha} .
$$

Finally consider the Dirichlet forms $\mathscr{E}$ and $\mathscr{E}_{V}$ on $L^{2}\left(\mathbb{R}^{d}\right)$ and $L^{2}(V)$ :

$$
\mathscr{E}(\phi, \phi)=\frac{1}{2} \int_{\mathbb{R}^{d}} \int_{\mathbb{R}^{d}}(\phi(x)-\phi(y))^{2} g_{\alpha}^{-1}(x-y) d x d y, \quad \mathscr{E}_{V}(\phi, \phi)=\sum_{n} \frac{1}{\lambda_{n}}\left\langle\phi, e_{n}\right\rangle_{V}^{2}
$$

Let $\mathscr{G}, \mathscr{G}_{V}$ be the extended domains of $\mathscr{E}$ and $\mathscr{E}_{V}$. Both Dirichlet forms are regular, cf. Example 1.5.1 of [7]. $\mathscr{E}$ is the Dirichlet form associated with the symmetric $\alpha$-stable process on $\mathbb{R}^{d}$, whereas $\mathscr{E}_{V}$ is the Dirichlet form of the symmetric $\alpha$-stable process imbedded in the unit cube $V$. Using the positivity and continuity of $K_{V}$ on $L^{2}(V)$, we have, for each dense subset $\mathscr{D}(V)$ of $L^{2}(V)$,

$$
\begin{aligned}
\mathscr{E}_{V}(\phi, \phi) & =\sup \left\{2\langle\phi, f\rangle_{V}-\left\langle f, K_{V} f\right\rangle_{V}: f \in \mathscr{D}(V)\right\} \\
& =\sup \left\{\frac{\langle\phi, f\rangle_{V}^{2}}{\left\langle f, K_{V} f\right\rangle_{V}}: f \in \mathscr{D}(V)\right\}
\end{aligned}
$$

\section{Lemma A.7.}

$$
\mathscr{E}_{V}\left(1_{V}, 1_{V}\right)=\inf \left\{\mathscr{E}(h, h): h \in C^{1}\left(\mathbb{R}^{d}\right) \cap L^{2}\left(\mathbb{R}^{d}\right), h \geqq 0, h=1 \text { on } V\right\}=\operatorname{cap}_{\alpha}(V)
$$

Proof. By Lemma 3.1.1, Problem 3.3.2 and Example 3.3.1 of [7], (see also [10], Theorem 2.3, page 138)

$$
\begin{aligned}
\operatorname{cap}_{\alpha}(V) & \equiv \inf \{\mathscr{E}(h, h): h \in \mathscr{G}, h \geqq 0, h=1 \text { on } V\} \\
& =\inf \left\{\mathscr{E}(h, h): h \in C^{1}\left(\mathbb{R}^{d}\right) \cap L^{2}\left(\mathbb{R}^{d}\right), h \geqq 0, h=1 \text { on } V\right\} .
\end{aligned}
$$


Also

$$
\operatorname{cap}_{\alpha}(V)=\sup \left\{2 \mu(V)-\int_{V} \int_{V} g_{\alpha}(x-y) \mu(d x) \mu(d y):\right.
$$

$\mu$ positive Radon measure on $V$ with finite energy $\}$.

For each $\mu$ with finite energy, we can find a sequence $\left\{f_{n}\right\} \subseteq L^{2}(V)$, such that

$$
\lim _{n \rightarrow \infty} 2\left\langle I_{V}, f_{n}\right\rangle_{V}-\left\langle f_{n}, K_{V} f_{n}\right\rangle_{V}=2 \mu(V)-\int_{V} \int_{V} g_{\alpha}(x-y) \mu(d x) \mu(d y),
$$

cf. Example 3.2.1 of [7]. Thus, by (A.6)

$$
\operatorname{cap}_{\alpha}(V)=\sup \left\{2\left\langle 1_{V}, f\right\rangle_{V}-\left\langle f, K_{V} f\right\rangle_{V}: f \in L^{2}(V)\right\}=\mathscr{E}_{V}\left(1_{V}, 1_{V}\right) .
$$

Lemma A.10. Let $f$ be Riemann integrable on $V$ and let $h \in C^{1}\left(\mathbb{R}^{d}\right) \cap L^{2}\left(\mathbb{R}^{d}\right)$. Set $f_{N}(k)=f(k / N), h_{N}(k)=h(k / N)$, then

$$
\begin{aligned}
& \lim _{N \rightarrow \infty} N^{-d-x}\left\langle f_{N}, G_{N} f_{N}\right\rangle_{V_{N}}=\left\langle f, K_{V} f\right\rangle_{V}, \\
& \lim _{N \rightarrow \infty} N^{-d+x}\left\langle h_{N}, G^{-1} h_{N}\right\rangle_{\mathbb{Z}^{d}}=\mathscr{E}(h, h) .
\end{aligned}
$$

Proof. This follows from Lemma A.2 and Riemann integration:

$$
\begin{aligned}
& \lim _{N \rightarrow \infty} N^{-d-\alpha}\left\langle f_{N}, G_{N} f_{N}\right\rangle_{V_{N}} \\
& =\lim _{M \rightarrow \infty} \lim _{N \rightarrow \infty} \sum_{k, j \in V_{N},|j-k|>M} f(k \mid N) N^{d-\alpha} G(k-j) f(j / N) N^{-2 d} \\
& =\lim _{N \rightarrow \infty} \sum_{k, j \in V_{N}, j \neq k} f(k / N) g_{x}(j / N-k / N) f(j / N) N^{-2 d}=\left\langle f, K_{V} f\right\rangle_{V},
\end{aligned}
$$

and

$$
\begin{aligned}
& \lim _{N \rightarrow \infty} N^{-d+\alpha}\left\langle h_{N}, G^{-1} h_{N}\right\rangle_{\mathbb{Z}^{d}} \\
& =\frac{1}{2} \lim _{M \rightarrow \infty} \lim _{N \rightarrow \infty} \sum_{k, j \in \mathbb{Z}^{d},|j-k|>M}(h(k / N)-h(j / N))^{2} N^{d+\alpha} Q(k-j) N^{-2 d} \\
& =\frac{1}{2} \lim _{N \rightarrow \infty} \sum_{k, j \in \mathbb{Z}^{d}, j \neq k}(h(k / N)-h(j / N))^{2} g_{\alpha}^{-1}(j / N-k / N) N^{-2 d} \\
& =\mathscr{E}(h, h) . \quad \square
\end{aligned}
$$

Proposition A.11. Let $f \in C^{1}(V)$. Set $f_{N}(k)=f(k / N)$, then

$$
\lim _{N \rightarrow \infty} N^{-d+\alpha}\left\langle f_{N}, G_{N}^{-1} f_{N}\right\rangle_{V_{N}}=\mathscr{E}_{V}(f, f) \text {. }
$$

In particular if $f=1_{V}$, then $f_{N}=1_{V_{N}}$ and

$$
\lim _{N \rightarrow \infty} N^{-d+x}\left\langle 1_{V_{N}}, G_{N}^{-1} 1_{V_{N}}\right\rangle_{V_{N}}=\lim _{N \rightarrow \infty} N^{-d+x} \operatorname{cap}_{N}\left(V_{N}\right)=\operatorname{cap}_{\alpha}(V)
$$

Proof. First note that for any $\phi \in C(V)$,

$$
N^{-d+x}\left\langle f_{N}, G_{N}^{-1} f_{N}\right\rangle_{V_{N}} \geqq N^{-d} 2\left\langle f_{N}, \phi_{N}\right\rangle_{V_{N}}-N^{-d-x}\left\langle\phi_{N}, G_{N} \phi_{N}\right\rangle_{V_{N}} .
$$


Thus in view of Lemma A.10 and (A.6),

$$
\liminf _{N \rightarrow \infty} N^{-d+\alpha}\left\langle f_{N}, G_{N}^{-1} f_{N}\right\rangle_{V_{N}} \geqq \sup _{\phi \in C(V)}\left\{2\langle f, \phi\rangle_{V}-\left\langle\phi, K_{V} \phi\right\rangle_{V}\right\}=\mathscr{E}_{V}(f, f) .
$$

Next for each $h \in C^{1}\left(\mathbb{R}^{d}\right) \cap L^{2}\left(\mathbb{R}^{d}\right)$, with $h=f$ on $V$

$$
\begin{aligned}
\left\langle f_{N}, G_{N}^{-1} f_{N}\right\rangle_{V_{N}} & =\sup _{\phi_{N} \in L^{2}\left(V_{N}\right)}\left\{2\left\langle h_{N}, \phi_{N}\right\rangle_{V_{N}}-\left\langle\phi_{N}, G_{N \phi N}\right\rangle_{V_{N}}\right\} \\
& \leqq \sup _{\phi \in L^{2}\left(\mathbb{Z}^{d}\right)}\left\{2\left\langle h_{N}, \phi\right\rangle_{\mathbb{Z}^{d}}-\langle\phi, G \phi\rangle_{\mathbb{Z}^{d}}\right\} \\
& =\left\langle h_{N}, G^{-1} h_{N}\right\rangle_{\mathbb{Z}^{d}}=\frac{1}{2} \sum_{j, k \in \mathbb{Z}^{d}}(h(j / N)-h(k / N))^{2} Q(j-k) .
\end{aligned}
$$

Using Lemmas A.7 and A.10, we see that

$$
\begin{aligned}
\limsup _{N \rightarrow \infty} N^{-d+x}\left\langle f_{N}, G_{N}^{-1} f_{N}\right\rangle_{V_{N}} & \leqq \inf \left\{\mathscr{E}(h, h): h \in C^{1}\left(\mathbb{R}^{d}\right) \cap L^{2}\left(\mathbb{R}^{d}\right), h=f \text { on } V\right\} \\
& =\mathscr{E}_{V}(f, f) .
\end{aligned}
$$

Finally, let $\left\{\xi_{n}=Y_{1}+\ldots+Y_{n}\right\}$ be a random walk generated by $Q$ and $\tau=$ $\inf \left\{n \geqq 0: \xi_{n} \in L \mathbb{Z}^{d}\right\}$. Remember that, by the random walk representation, we have

$$
q^{L}(i, j)=\mathbb{P}_{i}\left(\xi_{\tau}=j\right), \quad G^{L}(i, j)=\mathbb{E}_{i}\left[\sum_{n=0}^{\tau-1} 1_{\xi_{n}=j}\right]
$$

Proposition A.12. Assume $\left(b^{\prime}\right)$, then there exists $c_{3}>0$, such that, for $|i-j|>1$,

$$
\begin{aligned}
G^{L}(i, j) & \leqq c_{3} L^{c_{4}}(\log |i-j|)^{d+2+\alpha}|i-j|^{-d-\alpha}, \\
q^{L}(i, j) & \leqq c_{3} L^{c_{4}}(\log |i-j|)^{d+2+\alpha}|i-j|^{-d-\alpha},
\end{aligned}
$$

with $c_{4}=(d+\alpha)(d+2+\alpha)$. Also, for each $i \in \Lambda$,

$$
\lim _{L \rightarrow \infty} G^{L}(i, i)=G(i, i) \text {. }
$$

Proof. First remark that, for $i \in \Lambda$,

$G(i, i)-G^{L}(i, i)=\mathbb{E}_{i}\left[\sum_{n=\tau}^{\infty} 1_{\{i\}}\left(\xi_{n}\right)\right]=\sum_{j \in L \mathbb{Z}^{d}} q^{L}(i, j) G(i, j) \leqq \max _{i \in \Lambda, j \in L \mathbb{Z}^{d}} G(i, j) \rightarrow 0$, as $L \rightarrow \infty$.

Next let $\tau_{j}=\inf \left\{n \geqq 0: \xi_{n}=j\right\}$, then, referring to the proof of Lemma A.7 in [1],

$$
\begin{gathered}
G^{L}(i, j) \leqq G(0,0)\left(\mathbb{P}_{i}\left(\tau_{j} \leqq T\right)+\mathbb{P}_{i}(\tau>T)\right) \\
\mathbb{P}_{i}\left(\xi_{\tau}=j\right) \leqq \mathbb{P}_{i}\left(\tau_{j} \leqq \tau\right) \leqq \mathbb{P}_{i}\left(\tau_{j} \leqq T\right)+\mathbb{P}_{i}(\tau>T)
\end{gathered}
$$

for any $T \geqq 1$. We claim that

$$
\begin{gathered}
\mathbb{P}_{i}(\tau>T) \leqq e^{-k_{1} T L^{-d-\alpha}}, \\
\mathbb{P}_{i}(\tau, \leqq T) \leqq k_{2} T^{d+2+\alpha}|j-i|^{-d-\alpha},
\end{gathered}
$$


for some $k_{1}, k_{2}>0$. Equation (A.13) follows from these estimates by choosing $T=\frac{d+\alpha}{k_{1}} L^{d+\alpha} \log |j-i|$.

Proof of $(A .15)$. Simply note that

$$
\mathbb{P}_{i}(\tau>T)=\mathbb{P}_{i}\left(\xi_{0} \notin L \mathbb{Z}^{d}, \ldots, \xi_{T} \notin L \mathbb{Z}^{d}\right) \leqq\left(1-\rho_{L}\right)^{T},
$$

where

$$
\rho_{L}=\min _{i \in \mathbb{Z}^{d}} \mathbb{P}_{l}\left(\xi_{1} \in L \mathbb{Z}^{d}\right) \geqq \min _{l \in V_{L / 2}} Q(i, 0) \geqq k L^{-d-\alpha},
$$

for some $k>0$.

Proof of (A.16). The crucial step is to show that, for each $|j|>1$,

$$
\mathbb{P}_{0}\left(\xi_{n}=j\right) \leqq k_{3} n^{d+1+x}|j|^{-d-x} .
$$

Once (A.17) is shown, (A.16) follows from

$$
\mathbb{P}_{i}\left(\tau_{j} \leqq T\right) \leqq \sum_{n=1}^{T} \mathbb{P}_{0}\left(\xi_{n}=j-i\right) \leqq k_{4} T^{d+2+\alpha}|i-j|^{-d-\alpha} .
$$

In order to prove (A.17), note that,

$$
\begin{aligned}
\mathbb{P}_{0}\left(\xi_{n}=j\right) & =\mathbb{P}_{0}\left(\xi_{n}=j ; \bigcup_{l=1}^{n}\left\{\left|Y_{l}\right| \geqq \frac{|j|}{n}\right\}\right) \leqq n \mathbb{P}_{0}\left(\xi_{n}=j ;\left|Y_{n}\right| \geqq \frac{|j|}{n}\right) \\
& =n \sum_{\ell \in \mathbb{Z}^{d}} \mathbb{P}_{0}\left(Y_{n}=\ell ;\left|Y_{n}\right| \geqq \frac{|j|}{n}\right) \mathbb{P}_{0}\left(\xi_{n-1}=j-\ell\right),
\end{aligned}
$$

and use the fact that, for $\ell \geqq \frac{|j|}{n}$,

$$
\mathbb{P}_{0}\left(Y_{n}=\ell\right) \leqq k_{3}|j|^{-d-\alpha} n^{d+\alpha} .
$$

We conclude this Appendix with a proof of the hypercontractive estimate:

Proposition A.18. Let $\left\{\xi_{i}\right\}_{i \in \mathbb{Z}^{d}}$ be a Gaussian field of zero mean and summable covariance $R(i, j)=R(|i-j|)$. Assume that

$$
R(|i-j|) \leqq C|i-j|^{-d-\delta},
$$

for some $\delta, C>0$. Then there exists a constant $C_{R} \geqq 1$, independent of $N$, such that for any bounded measurable function $f(\cdot)$,

$$
E\left[\prod_{i \in V_{N}} f\left(\xi_{l}\right)\right] \leqq\|f\|_{C_{R}}^{\left|V_{N}\right|}
$$

where $\|f\|_{C_{R}}=\left(E\left[\left|f\left(\xi_{0}\right)\right|^{C_{R}}\right]\right)^{1 / C_{R}}$.

Proof. Let $I_{1}, I_{2}$ be two disjoint sets of indices in $V_{N}$. For any two vectors $\left\{\alpha_{i}\right\}_{i \in V_{N}},\left\{\beta_{i}\right\}_{l \in V_{N}}$, let $\langle\alpha, \beta\rangle=\sum_{i, j \in V_{N}} \alpha_{l} \beta_{j} R(i, j)$, with the obvious definition of $\|\alpha\|$. Following [2] (see p. 648, last line, and work in the time domain instead of in the frequency domain), let

$$
\tau_{\xi}^{I_{1}, I_{2}}=\sup \left\{\langle\alpha, \beta\rangle:\|\alpha\|=\|\beta\|=1, \alpha_{i}=0 \forall i \notin I_{1}, \beta_{i}=0 \forall i \notin I_{2}\right\} .
$$


By an adaptation of Nelson's hypercontractive estimates similar to [2], lemma, p. 645, for any two bounded functions $f_{1}, f_{2}$ measurable respectively on $\mathscr{F}_{I_{1}}=$ $\sigma\left(\xi_{i}: i \in I_{1}\right), \mathscr{F}_{I_{2}}=\sigma\left(\xi_{j}: j \in I_{2}\right)$,

$$
E\left[\left|f_{1}(\xi) f_{2}(\xi)\right|\right] \leqq\left\|f_{1}\right\|_{1+\tau_{\xi} I_{1}, I_{2}}\left\|f_{2}\right\|_{1+\tau_{\xi}^{I_{1}}, I_{2}} .
$$

Note that

$$
\begin{aligned}
& \langle\alpha, \beta\rangle=\left|\sum_{i \in I_{1}, j \in I_{2}} \alpha_{i} \beta_{j} R(i, j)\right| \leqq \sum_{i, j}\left(\alpha_{i}^{2}+\beta_{j}^{2}\right)|R(|i-j|)| \\
& \quad \leqq\|\alpha\|_{2}^{2}\|\beta\|_{2}^{2}\left(\sup _{i \in I_{1}} \sum_{j \in I_{2}}|R(|i-j|)|+\sup _{j \in I_{2}} \sum_{i \in I_{1}}|R(|i-j|)|\right),
\end{aligned}
$$

with $\|\alpha\|_{2}$ denoting the $\ell_{2}$ norm of $\alpha$. On the other hand,

$$
\|\alpha\|^{2}=\|\alpha\|_{2}^{2} R(0)+\sum_{i, j \in I_{1}} \alpha_{i} \alpha_{j} \bar{R}(|i-j|),
$$

where $\tilde{R}(x)=R(x)$ if $x \neq 0$ and $\tilde{R}(0)=0$. Hence,

$$
\|\alpha\|^{2} \geqq\|\alpha\|_{2}^{2}\left(1-2 \sup _{i \in I_{1}} \sum_{j \in I_{1}}|\bar{R}(|i-j|)|\right),
$$

with an analogous expression for $\|\beta\|$. It follows that

$$
\tau_{\tilde{\zeta}}^{I_{1}, I_{2}} \leqq 1 \wedge \frac{\left(\sup _{i \in I_{1}} \sum_{j \in I_{2}}|R(|i-j|)|+\sup _{j \in I_{2}} \sum_{i \in I_{1}}|R(|i-j|)|\right)}{\left(1-2 \sup _{i \in I_{1}} \sum_{j \in I_{1}}|\bar{R}(|i-j|)|\right)\left(1-2 \sup _{i \in I_{2}} \sum_{j \in I_{2}}|\bar{R}(|i-j|)|\right)} .
$$

Let now $V_{N}^{o}\left(V_{N}^{e}\right)$ denote the odd (respectively, even) points in $V_{N}$. Then, using the above,

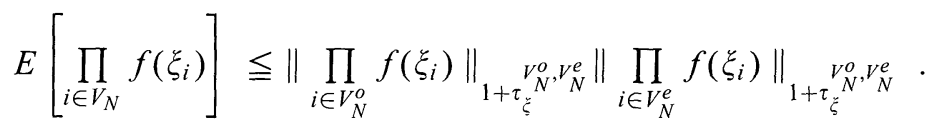

One may now iterate this inequality, partitioning in each stage the "odd" and the "even" parts again to two subsets. To keep track of the partitioning, we use the multi-index $\ell_{k} \in\{0,1\}^{k}$ to denote the partition history of each of the $2^{k}$ sets in the $k^{\text {th }}$ interation, denoted $V_{N}^{\ell_{k}, k}$, with 0 representing "odd" and 1 representing "even." Note that

$$
\left.\sup _{i \in V_{N}^{\ell_{k}, k}} \sum_{J \in V_{N}^{\ell_{k}, k}}|\bar{R}(|i-j|)|\right) \leqq \sum_{|j| \geqq 2\lfloor k / d\rfloor} R(|j|) \rightarrow_{k \rightarrow \infty} 0 .
$$

Let $\ell_{k}^{k-1}$ denote the truncation of the last coordinate in $\ell_{k}$. Using the above, it holds that for all $k>k_{0}$ (with $k_{0}$ depending on $R$ only), and all $\ell_{k}, \bar{\ell}_{k}$ satisfying $\ell_{k}^{k-1}=\bar{\ell}_{k}^{k-1}$,

$$
\tau_{\xi}^{V_{k}^{l_{k}, k}, V_{N}^{\bar{\ell}_{k}, k}} \leqq 4 \sum_{j \in(2\lfloor k / d\rfloor) V_{N}} \bar{R}(|j|)
$$


Let $\tau_{k}=4 \sum_{j \in 2(\lfloor k / d\rfloor)_{N}} \bar{R}(|j|)$. Iterating the basic inequality (A.20) yields now

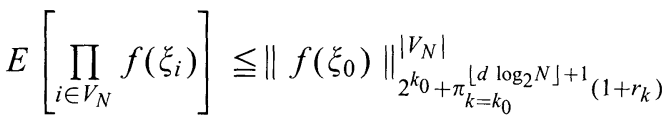

$$
\begin{aligned}
& \leqq\left\|f\left(\xi_{0}\right)\right\|_{2^{k_{0}+\pi_{k=k_{0}}\left(1+r_{k}\right)}}^{\left|V_{N}\right|}=\left\|f\left(\xi_{0}\right)\right\|_{C_{R}}^{\left|V_{N}\right|},
\end{aligned}
$$

where $C_{R}=2^{k_{0}}+\pi_{k=k_{0}}^{\infty}\left(1+r_{k}\right)<\infty$ and the last inequality follows from the fact that $\sum_{k=k_{0}}^{\infty} r_{k}<\infty$ due to (A.19).

Acknowledgements. We are grateful to J. Lebowitz and C. Maes for suggesting this problem and to R. Pemantle for the proof of (A.17).

\section{References}

0. Bolthausen, E., Deuschel, J.-D.: Critical large deviations for Gaussian fields in the phase transition regime. I. Ann. Proba. 21, 1876-1920 (1994)

1. Bricmont, J., El Mellouki, A., Fröhlich, J.: Random surfaces in statistical mechanics: roughening, rounding, wetting, J. Stat. Phys. 42, 743-798 (1986)

2. Chyonobu, T., Kusuoka, S.: The large deviation principle for hypermixing processes. Proba. Th. and Rel. Fields 78, 627-649 (1988)

3. Deuschel, J.-D., Stroock, D.W.: Large Deviations. Boston: Academic Press, 1989

4. Deuschel, J.-D., Stroock, D.W., Zessin, H.: Microcanonical distributions for lattice Gases. Commun. Math. Phys. 139, 83-101 (1991)

5. Dobrushin, R.L.: Prescribing a system of random variables by conditional distribution. Theor. Probab. Appl. 15, 458-486 (1970)

6. van Enter, A.C.D., Fernandez, R., Sokal, A.D.: Regularity properties and pathologies of position-space renormalization-group transformations: Scope and limitations of Gibbsian theory. J. Stat. Phys. 72, 879-1169 (1993)

7. Fukushima, M.: Dirichlet forms and Markov processes. Amsterdam: North-Holland, 1980

8. Georgii, H.-O.: Gibbs measures and phase transitions. Berlin: de Gruyter, 1988

9. Künsch, H.R.: Almost sure entropy and the variational principle for random fields with unbounded state space. Zeit. Wahr. Th. verw. Geb. 58, 69-85 (1981)

10. Landkof, N.S.: Foundations of Modern Potential Theory. Grundlehren 180, Berlin, Heidelberg, New York: Springer, 1972

11. Lebowitz, J.L., Maes, C.: The effect of an external field on an interface, entropy repulsion. J. Stat. Phys. 46, 39-49 (1987)

12. Le Gall, J.-F, Rosen, J.: The range of stable random walks. Ann. of Proba. 19, 650-705 (1991)

13. Spitzer, F.: Principles of random walks. 2nd ed. Berlin, Heidelberg, New York: Springer, 1976 
